\title{
Volterra operators and Hankel forms on Bergman spaces of Dirichlet series
}

\section{H. Bommier-Hato ${ }^{1}$}

Received: 15 January 2020 / Accepted: 16 February 2020 / Published online: 2 March 2020

(c) The Author(s) 2020

\section{Abstract}

For a Dirichlet series $g$, we study the Volterra operator $T_{g} f(s)=-\int_{s}^{+\infty} f(w) g^{\prime}(w)$ $d w$, acting on a class of weighted Hilbert spaces $\mathcal{H}_{w}^{2}$ of Dirichlet series. We obtain sufficient / necessary conditions for $T_{g}$ to be bounded (resp. compact), involving BMO and Bloch type spaces on some half-plane. We also investigate the membership of $T_{g}$ in Schatten classes. Moreover, we show that if $T_{g}$ is bounded, then $g$ is in $\mathcal{H}_{w}^{p}$, the $L^{p}$-version of $\mathcal{H}_{w}^{2}$, for every $0<p<\infty$. We also relate the boundedness of $T_{g}$ to the boundedness of a multiplicative Hankel form of symbol $g$, and the membership of $g$ in the dual of $\mathcal{H}_{w}^{1}$.

Keywords Volterra operator · Dirichlet series · Hankel forms

Mathematics Subject Classification Primary 31B10 - 32A36; Secondary 30B50 . $30 \mathrm{H} 20$

\section{Introduction}

Dirichlet series are functions of the form

$$
f(s)=\sum_{n=1}^{+\infty} a_{n} n^{-s}, \quad \text { with } s \in \mathbb{C} .
$$

Communicated by Adrian Constantin.

The author was supported by the FWF project P 30251-N35.

H. Bommier-Hato

helene.bommier@gmail.com

1 Faculty of Mathematics, University of Vienna, Oskar-Morgenstern-Platz 1, 1090 Vienna, Austria 
For a real number $\theta, \mathbb{C}_{\theta}$ stands for the half-plane $\{s, \Re s>\theta\}$, and $\mathbb{D}$ for the unit disk. $\mathcal{D}$ denotes the class of functions $f$ of the form (1.1) in some half-plane $\mathbb{C}_{\theta}$, and $\mathcal{P}$ is the space of Dirichlet polynomials.

The increasing sequence of prime numbers will be denoted by $\left(p_{j}\right)_{j \geq 1}$, and the set of all primes by $\mathbb{P}$. Given a positive integer $n, n=p^{\kappa}$ will stand for the prime number factorization $n=p_{1}^{\kappa_{1}} p_{2}^{\kappa_{2}} \cdots p_{d}^{\kappa_{d}}$, which associates uniquely to $n$ the finite multi-index $\kappa(n)=\left(\kappa_{1}, \kappa_{2}, \ldots, \kappa_{d}\right)$. The number of prime factors in $n$ is denoted by $\Omega(n)$ (counting multiplicities), and by $\omega(n)$ (without multiplicities).

The space of eventually zero complex sequences $c_{00}$ consists in all sequences which have only finitely many non zero elements. We set $\mathbb{D}_{\text {fin }}^{\infty}=\mathbb{D}^{\infty} \cap c_{00}$ and $\mathbb{N}_{0 \text {,fin }}^{\infty}=$ $\mathbb{N}_{0}^{\infty} \cap c_{00}$, where $\mathbb{N}_{0}=\mathbb{N} \cup\{0\}$ is the set of non-negative integers.

Let $F: \mathbb{D}_{\text {fin }}^{\infty} \rightarrow \mathbb{C}$ be analytic, i.e. analytic at every point $z \in \mathbb{D}_{\text {fin }}^{\infty}$ separately with respect to each variable. Then $F$ can be written as a convergent Taylor series

$$
F(z)=\sum_{\alpha \in \mathbb{N}_{0, \mathrm{fin}}^{\infty}} c_{\alpha} z^{\alpha}, z \in \mathbb{D}_{\mathrm{fin}}^{\infty}
$$

The truncation $A_{m} F$ of $F$ onto the first $m$ variables is defined by

$$
A_{m} F(z)=F\left(z_{1}, \ldots, z_{m}, 0,0, \ldots\right)
$$

For $z, \chi$ in $\mathbb{D}^{\infty}$, we set $z \cdot \chi:=\left(z_{1} \chi_{1}, z_{2} \chi_{2}, \ldots\right)$, and $\mathfrak{p}^{\mathbf{x}}:=\left(p_{1}^{x}, p_{2}^{x}, \ldots\right)$ for a real number $x$, .

The Bohr lift [11] of the Dirichlet series $f(s)=\sum_{n=1}^{+\infty} a_{n} n^{-s}$ is the power series

$$
\mathcal{B} f(\chi)=\sum_{n=1}^{+\infty} a_{n} \chi^{\kappa(n)}=\sum_{\alpha \in \mathbb{N}_{0, \mathrm{fin}}^{\infty}} \tilde{a}_{\alpha} \chi^{\alpha}, \text { where } \tilde{a}_{\alpha}=a_{p^{\alpha}}, \chi \in \mathbb{D}_{\mathrm{fin}}^{\infty}
$$

with the multiindex notation $\chi^{\alpha}=\chi_{1}^{\alpha_{1}} \chi_{2}^{\alpha_{2}} \cdots$.

Given a sequence of positive numbers $w=\left(w_{n}\right)_{n}=(w(n))_{n}$, one considers the Hilbert space (see [21,23])

$$
\mathcal{H}_{w}^{2}:=\left\{\sum_{n=1}^{+\infty} a_{n} n^{-s}: \sum_{n=1}^{+\infty} \frac{\left|a_{n}\right|^{2}}{w_{n}}<+\infty\right\} .
$$

The choice $w_{n}=1$ corresponds to the space $\mathcal{H}^{2}$, introduced in [19].

The weights considered in this article satisfy $w_{n}=O\left(n^{\epsilon}\right)$ for every $\epsilon>0$; from the Cauchy-Schwarz inequality, Dirichlet series in $\mathcal{H}_{w}^{2}$ absolutely converge in $\mathbb{C}_{1 / 2}$.

We are interested in the Volterra operator $T_{g}$ of symbol $g(s)=\sum_{n=1}^{+\infty} b_{n} n^{-s}$, defined by

$$
T_{g} f(s):=-\int_{s}^{+\infty} f(w) g^{\prime}(w) d w, \Re s>\frac{1}{2} .
$$


On the unit disk $\mathbb{D}$, the Volterra operator, whose symbol is an analytic function $g$, is given by

$$
J_{g} f(z):=\int_{0}^{z} f(u) g^{\prime}(u) d u, z \in \mathbb{D}
$$

Pommerenke [26] showed that $J_{g}(1.3)$ is bounded on the Hardy space $H^{2}(\mathbb{D})$ if and only if $g$ is in $B M O A(\mathbb{D})$. Let $\sigma$ be the Haar measure on the unit circle $\mathbb{T}$. Fefferman's duality Theorem states that $B M O A(\mathbb{D})$ is the dual space of $H^{1}(\mathbb{D})$. Thus the boundedness of $J_{g}$ is equivalent to the boundedness of the Hankel form

$$
H_{g}(f, h):=\int_{\mathbb{T}} f(u) h(u) \overline{g(u)} d \sigma(u), f, h \in H^{2}(\mathbb{D}) .
$$

Let $V$ be the Lebesgue measure on $\mathbb{C}$, normalized such that $V(\mathbb{D})=1$.

Many authors, in particular [2], have studied Volterra operators on Bergman spaces of $\mathbb{D}$. The classical Bergman space $A_{\gamma}^{2}(\mathbb{D}), \gamma>0$, is associated to the measure $d \tilde{m}_{\gamma}(z):=\gamma\left(1-|z|^{2}\right)^{\gamma-1} d V(z) . J_{g}$ is bounded on $A_{\gamma}^{2}(\mathbb{D})$ if and only if $g$ is in the Bloch space, which is the dual of $A_{\gamma}^{1}(\mathbb{D})$.

The Bergman space of the finite polydisk $A_{\gamma}^{2}\left(\mathbb{D}^{d}\right), d \geq 1$, corresponds to the measure

$$
d \widetilde{v}_{\gamma}(z):=d \tilde{m}_{\gamma}\left(z_{1}\right) \times \cdots \times d \tilde{m}_{\gamma}\left(z_{d}\right)
$$

The boundedness of the Hankel form

$$
H_{g}(f, h):=\int_{\mathbb{D}^{d}} f(z) h(z) \overline{g(z)} d \widetilde{\nu}_{\gamma}(z), f, h \in A_{\gamma}^{2}\left(\mathbb{D}^{d}\right),
$$

is equivalent to the membership of $g$ to the Bloch space (see [17]), defined by

$\operatorname{Bloch}\left(\mathbb{D}^{d}\right):=\left\{f: \mathbb{D}^{d} \rightarrow \mathbb{C}\right.$ holomorphic : $\left.\max _{\kappa \in \mathcal{I}_{d}} \sup _{z \in \mathbb{D}^{d}}\left|\partial^{\kappa} f(\kappa . z)\right|(1-|z|)^{\kappa}<+\infty\right\}$,

where $\mathcal{I}_{d}$ denotes the set of multi-indices $\kappa=\left(\kappa_{1}, \ldots, \kappa_{d}\right)$, with entries in $\{0,1\}$, and

$$
z=\left(z_{1}, \ldots, z_{d}\right), \partial^{\kappa}=\partial_{z_{1}}^{\kappa_{1}} \cdots \partial_{z_{d}}^{\kappa_{d}},(1-|z|)^{\kappa}=\left(1-\left|z_{1}\right|\right)^{\kappa_{1}} \cdots\left(1-\left|z_{d}\right|\right)^{\kappa_{d}}
$$

Recall that for $0<p<\infty$, the Hardy space of Dirichlet series $\mathcal{H}^{p}$ is the space of Dirichlet series $f \in \mathcal{D}$ such that $\mathcal{B} f$ is in $H^{p}\left(\mathbb{D}^{\infty}\right)$, endowed with the norm

$$
\|f\|_{\mathcal{H}^{p}}:=\|\mathcal{B} f\|_{H^{p}\left(\mathbb{D}^{\infty}\right)}=\left(\int_{\mathbb{T}^{\infty}}|\mathcal{B} f(z)|^{p} d \sigma_{\infty}(z)\right)^{1 / p},
$$

$\sigma_{\infty}$ being the Haar measure of the infinite polytorus $\mathbb{T}^{\infty}$. 
The norm in the space $\mathcal{H}^{\infty}:=H^{\infty}\left(\mathbb{C}_{0}\right) \cap \mathcal{D}$ is

$$
\|f\|_{\mathcal{H}^{\infty}}=\sup _{s \in \mathbb{C}_{0}}|f(s)|
$$

Let $H^{\infty}\left(\mathbb{D}^{\infty}\right)$ be the space of series $F$ which are finitely bounded, i.e.

$$
\|F\|_{H^{\infty}(\mathbb{D} \infty)}=\sup _{m \in \mathbb{N}_{0}, z \in \mathbb{D}^{\infty}}\left|A_{m} F(z)\right|<\infty .
$$

Via the Bohr isomorphism, we have [16,19]

$$
\|f\|_{\mathcal{H}^{\infty}}=\|\mathcal{B} f\|_{H^{\infty}\left(\mathbb{D}^{\infty}\right)}
$$

Several abscissae are related to a function $g$ in $\mathcal{D}$, of the form $g(s)=\sum_{n=1}^{+\infty} b_{n} n^{-s}$ :

the abscissa of convergence $\sigma_{c}=\inf \left\{\sigma \in \mathbb{R}: \sum_{n=1}^{+\infty} b_{n} n^{-\sigma}\right.$ converges $\}$;

the abscissa of absolute convergence $\sigma_{a}=\inf \left\{\sigma \in \mathbb{R}: \sum_{n=1}^{+\infty}\left|b_{n}\right| n^{-\sigma}\right.$ converges $\}$; the abscissa of uniform convergence

$\sigma_{u}=\inf \left\{\theta \in \mathbb{R}: \sum_{n=1}^{+\infty} b_{n} n^{-s}\right.$ converges uniformly in $\left.\mathbb{C}_{\theta}\right\}$.

The abscissa of regularity and boundedness, denoted by $\sigma_{b}$, is the infimum of those $\theta$ such that $g(s)$ has a bounded analytic continuation, to the half-plane $\Re(s)>\theta+\epsilon$, for every $\epsilon>0$.

We have $-\infty \leq \sigma_{c} \leq \sigma_{u} \leq \sigma_{a} \leq+\infty$, and, if any of the abscissae is finite $\sigma_{a}-\sigma_{c} \leq 1$. Moreover, it is known that $\sigma_{b}=\sigma_{u}[11]$, and $\sigma_{a}-\sigma_{u} \leq \frac{1}{2}$.

Volterra operators (1.2) on the spaces $\mathcal{H}^{p}$ have been investigated in [13]. Our aim is to study similar questions for the spaces $\mathcal{H}_{w}^{2}$, associated to specific weights $w$ in the class $\mathcal{W}$ defined below.

Definition 1 Let $\beta>0$. A sequence $w$ belongs to $\mathcal{W}$ if it has one of the following forms:

(1) $w_{n}=[d(n)]^{\beta}$, where $d(n)$ is the number of divisors of the integer $n$. Then $\mathcal{H}_{w}^{2}:=\mathcal{B}_{\beta}^{2}$.

(2) $w_{n}=d_{\beta+1}(n)$, where $d_{\gamma}(n)$ are the Dirichlet coefficients of the power of the Riemann zeta function, namely $\zeta^{\gamma}(s)=\sum_{n=1}^{+\infty} d_{\gamma}(n) n^{-s}$. Then $\mathcal{H}_{w}^{2}:=\mathcal{A}_{\beta}^{2}$.

As in the case of $\mathcal{H}^{2}$ [13], we obtain sufficient/necessary conditions for $T_{g}$ to be bounded on the Hilbert spaces $\mathcal{H}_{w}^{2}$. However, due to the lack of information of the behavior of the symbols in the strip $0<\Re s<1 / 2$, it seems difficult to get an " if and only if" condition. In the Hardy space setting, it is shown that $T_{g}$ is bounded on $\mathcal{H}^{2}$ provided that $g$ in $B M O A\left(\mathbb{C}_{0}\right)$. Since the spaces $\mathcal{A}_{\beta}^{2}$ and $\mathcal{B}_{\beta}^{2}$ (see Sect. 2) locally behave like Bergman spaces of the half plane $\mathbb{C}_{0}$, we would expect that the membership of $g$ in $\operatorname{Bloch}\left(\mathbb{C}_{0}\right)\left(\right.$ resp. Bloch $\left.\left(\mathbb{C}_{0}\right)\right)$ would imply the boundedness (resp. 
compactness) of $T_{g}$ on $\mathcal{H}_{w}^{2}$. We obtain such a sufficient condition when $\mathcal{B} g$ depends on a finite number of variables $z_{1}, \ldots, z_{d}$. However, our method specfically uses that $d$ is finite, and we do not know whether the same result holds if $\mathcal{B} g$ is a function of infinitely many variables.

Le $\mathfrak{N}_{d}$ be the set of positive integers which are multiples of the primes $p_{1}, \ldots, p_{d}$,

$$
\mathcal{D}_{d}:=\left\{f \in \mathcal{D}: f(s)=\sum_{n \in \mathfrak{N}_{d}} a_{n} n^{-s}\right\}, \text { and } \mathcal{H}_{d, w}^{p}:=\mathcal{H}_{w}^{p} \cap \mathcal{D}_{d}
$$

One of our main results is the following.

Theorem 1 Let $T_{g}$ be the operator defined by (1.2) for some Dirichlet series $g$ in $\mathcal{D}$.

(a) If $g(s)=\sum_{n=2}^{+\infty} b_{n} n^{-s}$ is in $\mathcal{D}_{d} \cap$ Bloch $\left(\mathbb{C}_{0}\right)$, then $T_{g}$ is bounded on $\mathcal{H}_{w}^{2}$ and

$$
\left\|T_{g}\right\|_{\mathcal{L}\left(\mathcal{H}_{w}\right)} \lesssim\|g\|_{B \operatorname{Bloch}\left(\mathbb{C}_{0}\right)}
$$

(b) If $g$ is in BMOA( $\left.\mathbb{C}_{0}\right)$, then $T_{g}$ is bounded on $\mathcal{H}_{w}^{2}$ and

$$
\left\|T_{g}\right\|_{\mathcal{L}\left(\mathcal{H}_{w}\right)} \lesssim\|g\|_{B M O A\left(\mathbb{C}_{0}\right)}
$$

(c) If $T_{g}$ is bounded on $\mathcal{H}_{w}^{2}$, then $g$ is in Bloch $\left(\mathbb{C}_{1 / 2}\right)$ and

$$
\|g\|_{\text {Bloch }\left(\mathbb{C}_{1 / 2}\right)} \lesssim\left\|T_{g}\right\|_{\mathcal{L}\left(\mathcal{H}_{w}\right)} .
$$

Via the Bohr lift, $\mathcal{H}_{w}^{2}$ are $L^{2}$-spaces of functions on the polydisk $\mathbb{D}^{\infty}$. Precisely, there exists a probability measure $\mu_{w}$ on $\mathbb{D}^{\infty}$ such that

$$
\|f\|_{\mathcal{H}_{w}^{2}}^{2}=\int_{\mathbb{D}^{\infty}}|\mathcal{B} f(z)|^{2} d \mu_{w}(z)
$$

Analogously to the spaces $\mathcal{H}^{p}$, we define the space $\mathcal{H}_{w}^{p}, 0<p<\infty$ (see Sect. 2), as the closure of Dirichlet polynomials under the norm (quasi-norm if $0<p<1$ )

$$
\|f\|_{\mathcal{H}_{w}^{p}}=\|\mathcal{B} f\|_{L^{p}\left(\mathbb{D}^{\infty}, \mu_{w}\right)} .
$$

Let $\mathcal{X}_{w}=\mathcal{X}\left(\mathcal{H}_{w}^{2}\right)$ be the space of symbols $g$ giving rise to bounded operators $T_{g}$ on $\mathcal{H}_{w}^{2}$. Our study provides the following strict inclusions:

$$
\operatorname{BMOA}\left(\mathbb{C}_{0}\right) \cap \mathcal{D} \subset_{\neq} \mathcal{X}_{w} \subset_{\neq} \cap_{0<p<\infty} \mathcal{H}_{w}^{p}
$$

We will also compare $\mathcal{X}_{w}$ with other spaces of Dirichlet series, in particular with the dual of $\mathcal{H}_{w}^{1}$, and the space of symbols $g$ generating a bounded Hankel form

$$
H_{g}(f h):=\langle f h, g\rangle_{\mathcal{H}_{w}^{2}}
$$


on the weak product $\mathcal{H}_{w}^{2} \odot \mathcal{H}_{w}^{2}$. As in the case of $\mathcal{H}^{2}$ [13], we only get partial results. For Dirichlet series involving $d$ primes, we have

$$
\mathcal{D}_{d} \cap \operatorname{Bloch}\left(\mathbb{C}_{0}\right) \subset \mathcal{D}_{d} \cap \mathcal{X}_{w} \subset \neq \mathcal{B}^{-1} \operatorname{Bloch}\left(\mathbb{D}^{d}\right) .
$$

The paper is organized as follows. Section 2 starts by presenting some properties of the spaces $\mathcal{H}_{w}^{2}$. As a space of analytic functions on the half-plane $\mathbb{C}_{1 / 2}, \mathcal{H}_{w}^{2}$ is continuously embedded in a space of Bergman type of $\mathbb{C}_{1 / 2}$. In view of the Bohr lift, the norm of $\mathcal{H}_{w}^{2}$ can be expressed in terms of a probability measure $\mu_{w}$ on the polydisk. For $0<p<\infty$, we consider the Bohr-Bergman space $\mathcal{H}_{w}^{p}$, and derive equivalent norms for these spaces.

In Sect. 3, we present some properties of the Dirichlet series which belong to a $\mathrm{BMO}$ or Bloch space of some half-plane $\mathbb{C}_{\theta}$. In particular, we relate the Carleson measures for both spaces of Dirichlet series and Bergman type spaces.

Section 4 is devoted to the proof of Theorem 1. First we consider the case when $g$ is a function of $p_{1}^{-s}, \ldots, p_{d}^{-s}$. To prove $(b)$, we observe that the boundedness of $T_{g}$ on $\mathcal{H}^{2}$ implies the boundedness of $T_{g}$ on $\mathcal{H}_{w}^{2}$. On another hand, combining the fact that $\mathcal{H}_{w}^{2}$ is embedded in a Bergman type space of the half-plane $\mathbb{C}_{1 / 2}$ with some characterizations of Carleson measures, we establish that

$$
\mathcal{X}_{w} \subset \operatorname{Bloch}\left(\mathbb{C}_{1 / 2}\right)
$$

Compactness and Schatten classes are considered in Sects. 5 and 6.

In Sect. 7, we consider some specific symbols: fractional primitives of translates of a "weighted zeta"-function and homogeneous symbols. These examples will be used in Sect. 8.

In Sect. 8, we investigate the relationship between the boundedness of the Volterra operator $T_{g}$, the boundedness of the Hankel form

$$
H_{g}(f h)=\langle f h, g\rangle_{\mathcal{H}_{w}^{2}}
$$

and the membership of $g$ in the dual of $\mathcal{H}_{w}^{1}$. In particular, we study examples of Hankel forms on Bergman spaces of Dirichlet series, which are the counterparts of the Hilbert multiplicative matrix [12].

Additionally, we show the strictness of the inclusions derived previously

$$
\operatorname{BMOA}\left(\mathbb{C}_{0}\right) \cap \mathcal{D} \subset_{\neq} \mathcal{X}_{w} \subset_{\neq} \cap_{0<p<\infty} \mathcal{H}_{w}^{p}
$$

and compare the space $\mathcal{D}_{d} \cap \mathcal{X}_{w}$ with Bloch spaces.

For two functions $f, g$, the notation $f=O(g)$ or $f \lesssim g$, means that there exists a constant $C$ such that $f \leq C g$. If $f=O(g)$ and $g=O(f)$, we write $f \asymp$ $g$. 


\section{The Bohr-Bergman spaces $\mathcal{B}_{\beta^{\prime}}^{2} \mathcal{A}_{\beta}^{2}$}

\subsection{The spaces $\mathcal{B}_{\beta^{\prime}}^{2}, \mathcal{A}_{\beta}^{2}$}

These spaces are related to number theory. The number of divisors of the integer $n$, $d(n)$, is $d(n)=\left(\kappa_{1}+1\right) \cdots\left(\kappa_{d}+1\right)$ when $n=p^{\kappa}$. We consider the following scale of Hilbert spaces

$$
\mathcal{B}_{\beta}^{2}=\left\{f(s)=\sum_{n=1}^{+\infty} a_{n} n^{-s}:\|f\|_{\mathcal{B}_{\beta}^{2}}:=\left(\sum_{+\infty}^{n=1} \frac{\left|a_{n}\right|^{2}}{[d(n)]^{\beta}}\right)^{\frac{1}{2}}<\infty\right\}, \text { for } \beta>0 .
$$

The case $\beta=0$ corresponds to the Hardy space $\mathcal{H}^{2}$. The reproducing kernels of $\mathcal{B}_{\beta}^{2}$ are

$$
K^{\mathcal{B}_{\beta}^{2}}(s, u)=\zeta_{\beta}(s+\bar{u}), \text { where } \zeta_{\beta}(s)=\sum_{n=1}^{+\infty}[d(n)]^{\beta} n^{-s}
$$

It is shown in [30] that there exists $\phi_{\beta}(s)$, an Euler product which converges absolutely in $\mathbb{C}_{1 / 2}$, such that

$$
\zeta_{\beta}(s)=[\zeta(s)]^{2^{\beta}} \phi_{\beta}(s) \text {, and } \phi_{\beta}(1) \neq 0 .
$$

Another family of spaces arises from the so-called generalized divisor function. For $\gamma>0$, the numbers $d_{\gamma}(n)$ are defined by the relation

$$
\zeta^{\gamma}(s)=\sum_{n=1}^{+\infty} d_{\gamma}(n) n^{-s}
$$

A computation involving Euler products shows that we have

$$
d_{\gamma}\left(p^{r}\right)=\frac{\gamma(\gamma+1) \cdots(\gamma+r-1)}{r !}, \text { for } p \in \mathbb{P}, \text { and any integer } r .
$$

From its definition, $d_{\gamma}$ is a multiplicative function, i.e. $d_{\gamma}(k l)=d_{\gamma}(k) d_{\gamma}(l)$ if $k$ and $l$ are relatively prime. Thus, $d_{\gamma}(n)$ can be computed explicitly from the decomposition $n=p^{\kappa}$.

We define the spaces

$$
\mathcal{A}_{\beta}^{2}=\left\{f(s)=\sum_{n=1}^{+\infty} a_{n} n^{-s}:\|f\|_{\mathcal{A}_{\beta}^{2}}:=\left(\sum_{+\infty}^{n=1} \frac{\left|a_{n}\right|^{2}}{d_{\beta+1}(n)}\right)^{\frac{1}{2}}<\infty\right\}, \text { for } \beta>0,
$$


with reproducing kernels $K^{\mathcal{A}_{\beta}^{2}}(s, u)=\zeta^{\beta+1}(s+\bar{u})$.

Notice that, in each case, the reproducing kernel has the form

$$
K^{\mathcal{H}_{w}^{2}}(s, u)=Z_{w}(s+\bar{u})
$$

where $Z_{w}(s):=\sum_{n=1}^{+\infty} w_{n} n^{-s}$ has a singularity at $s=1$, with an estimate of the type

$$
Z_{w}(s)=C_{w}(s-1)^{-(\delta+1)}[1+O(1)] .
$$

\subsection{Bohr-Bergman spaces on $\mathbb{D}^{\infty}$}

The Bohr correspondence is an isometry between $\mathcal{H}_{w}^{2}$ and the weighted Bergman space of the infinite polydisk

$$
H_{w}^{2}\left(\mathbb{D}^{\infty}\right)=\left\{\sum_{\nu \in \mathbb{N}_{0, \text { fin }}^{\infty}} a_{\nu} z^{v}: \sum_{\nu} \frac{\left|a_{v}\right|^{2}}{w_{v}}<\infty\right\}, \text { where } w_{\nu}=\prod_{j} w_{\nu_{j}}
$$

In particular, the space $\mathcal{H}^{2}$ is identified with the Hardy space $H^{2}\left(\mathbb{T}^{\infty}\right)$ [19].

Let us consider the following probability measures on the unit disk $\mathbb{D}$,

$$
\begin{aligned}
d m_{w}(z) & :=M\left(|z|^{2}\right) d V(z), \\
\text { where } M(r) & = \begin{cases}\frac{1}{\Gamma(\beta)}\left(\log \frac{1}{r}\right)^{\beta-1}, & \text { if } w_{n}=[d(n)]^{\beta}, \\
\beta(1-r)^{\beta-1}, & \text { if } w_{n}=d_{\beta+1}(n)\end{cases}
\end{aligned}
$$

On the finite polydisk $\mathbb{D}^{d}(d \in \mathbb{N})$, the corresponding Bergman spaces $H_{w}^{2}\left(\mathbb{D}^{d}\right)$ specifically $B_{\beta}^{2}\left(\mathbb{D}^{d}\right)$ and $A_{\beta}^{2}\left(\mathbb{D}^{d}\right)$ - are the $L^{2}$-closures of polynomials with respect to the norm

$$
\|f\|_{H_{w}^{2}\left(\mathbb{D}^{d}\right)}:=\left(\int_{\mathbb{D}^{d}}\left|f\left(z_{1}, \ldots, z_{d}\right)\right|^{2} d m_{w}\left(z_{1}\right) \times \cdots \times d m_{w}\left(z_{d}\right)\right)^{1 / 2}
$$

If $f(z)=\sum_{n \in \mathbb{N}^{d}} a_{n} z^{n}$ is defined on $\mathbb{D}^{d}$, we have

$$
\begin{aligned}
\|f\|_{B_{\beta}^{2}(\mathbb{D})}^{2} & =\sum_{n \in \mathbb{N}} \frac{\left|a_{n}\right|^{2}}{(n+1)^{\beta}} \\
\text { and }\|f\|_{A_{\beta}^{2}(\mathbb{D})}^{2} & =\sum_{n \in \mathbb{N}}\left|a_{n}\right|^{2} \frac{n !}{(\beta+1)(\beta+2) \cdots(\beta+n)} .
\end{aligned}
$$

When $d$ is finite, the estimate

$$
\frac{n !}{(\beta+1)(\beta+2) \cdots(\beta+n)} \asymp(1+n)^{-\beta}
$$


yields that, the spaces $B_{\beta}^{2}\left(\mathbb{D}^{d}\right)$ and $A_{\beta}^{2}\left(\mathbb{D}^{d}\right)$ coincide as sets, with equivalent norms. However, the norms are no longer equivalent in the case of infinitely many variables. The $\mathcal{H}_{w}^{2}$-norm will be computed via the rotation invariant probability measure

$$
d \mu_{w}(\chi)=d m_{w}\left(\chi_{1}\right) \times d m_{w}\left(\chi_{2}\right) \times d m_{w}\left(\chi_{3}\right) \times \cdots \text { on } \mathbb{D}^{\infty} .
$$

Applying the Bohr lift to a Dirichlet series $f(s)=\sum_{n=1}^{+\infty} a_{n} n^{-s}$, and using (2.2) for each variable, one obtains the following formula (see [5] in the case of $\mathcal{B}_{\beta}^{2}$ )

$$
\int_{\mathbb{D}^{\infty}}|\mathcal{B} f(\chi)|^{2} d \mu_{w}(\chi)=\sum_{n=1}^{+\infty} \frac{\left|a_{n}\right|^{2}}{w_{n}}=\|f\|_{\mathcal{H}_{w}^{2}}^{2}
$$

Definition 2 For $0<p<\infty$, the Bohr-Bergman spaces of Dirichlet series $\mathcal{B}_{\beta}^{p}$ and $\mathcal{A}_{\beta}^{p}$ - denoted by $\mathcal{H}_{w}^{p}$ - are the completions of the Dirichlet polynomials in the norm (quasi norm when $0<p<1$ )

$$
\|f\|_{\mathcal{H}_{w}^{p}}^{p}:=\int_{\mathbb{D}^{\infty}}|\mathcal{B} f(\chi)|^{p} d \mu_{w}(\chi)
$$

The Kronecker flow of the point $\chi=\left(\chi_{1}, \chi_{2}, \ldots\right) \in \mathbb{C}^{\infty}$ is given by

$$
\mathcal{T}_{t}(\chi)=\left(2^{-i t} \chi_{1}, 3^{-i t} \chi_{2}, 5^{-i t} \chi_{3}, \ldots\right), t \in \mathbb{R}
$$

which defines an ergodic flow on $\mathbb{T}^{\infty}$ by Kronecker's theorem.

Therefore, it follows from Fubini's Theorem that, for any rotation invariant probability measure $d \nu$ on $\mathbb{D}^{\infty}$ and any probability measure $d \lambda$ on $\mathbb{R}$, we have

$$
\|f\|_{L^{p}(\mathbb{D} \infty, d v)}^{p}=\int_{\mathbb{D}^{\infty}} \int_{\mathbb{R}}\left|(\mathcal{B} f)\left(\mathcal{T}_{t} \chi\right)\right|^{p} d \lambda(t) d \nu(\chi) .
$$

\subsection{On the half-plane $\mathbb{C}_{1 / 2}$}

For $\theta \in \mathbb{R}$, let $\tau_{\theta}$ be the following mapping from $\mathbb{D}$ to $\mathbb{C}_{\theta}$,

$$
\tau_{\theta}(z)=\theta+\frac{1+z}{1-z} .
$$

For $\delta>0$, the conformally invariant Bergman space $A_{i, \delta}\left(\mathbb{C}_{1 / 2}\right)$ is the space of those functions $f$ which are analytic in $\mathbb{C}_{1 / 2}$, and such that

$$
\|f\|_{A_{i, \delta}\left(\mathbb{C}_{1 / 2}\right)}^{2}:=\left\|f \circ \tau_{1 / 2}\right\|_{A_{\delta}^{2}(\mathbb{D})}^{2}=4^{\delta} \delta \int_{\mathbb{C}_{1 / 2}}|f(s)|^{2} \frac{\left(\sigma-\frac{1}{2}\right)^{\delta-1}}{\left|s+\frac{1}{2}\right|^{2 \delta+2}} d m(s)<\infty .
$$


The weights $w$ of the class $\mathcal{W}$ satisfy a Chebyshev-type estimate

$$
\sum_{n \leq x} w_{n} \asymp x(\log x)^{\delta}, \quad \text { where } \delta=\delta(w):= \begin{cases}2^{\beta}-1 & \text { if } w_{n}=[d(n)]^{\beta} \\ \beta & \text { if } w_{n}=d_{\beta+1}(n) .\end{cases}
$$

For any real number $\tau$, set $S_{\tau}=\left[\frac{1}{2}, 1\right] \times[\tau, \tau+1]$. As mentioned in the introduction, the Dirichlet series which belong the $\mathcal{H}_{w}^{2}$ absolutely converge in $\mathbb{C}_{1 / 2}$. The space $\mathcal{H}_{w}^{2}$ is locally embedded in $A_{i, \delta(w)}\left(\mathbb{C}_{1 / 2}\right)[23,25]$, which means

$$
\sup _{\tau \in \mathbb{R}} \int_{S_{\tau}}|f(s)|^{2} \frac{\left(\sigma-\frac{1}{2}\right)^{\delta-1}}{\left|s+\frac{1}{2}\right|^{2 \delta+2}} d m(s) \leq c\left(\mathcal{H}_{w}^{2}\right)\|f\|_{\mathcal{H}_{w}^{2}}^{2}
$$

Since functions in $\mathcal{H}_{w}^{2}$ are uniformly bounded in $\mathbb{C}_{1}$, these embeddings are global (see $[5,8])$.

Lemma 1 Let $\delta=\delta(w)$ be defined in (2.5). Then $\mathcal{H}_{w}^{2}$ is continuously embedded in $A_{i, \delta}\left(\mathbb{C}_{1 / 2}\right)$.

\subsection{Generalized vertical limits}

Every $\chi=\left(\chi_{1}, \chi_{2}, \ldots\right)$ in $\mathbb{C}^{\infty}$ defines a completely multiplicative function by the formula $\chi(n)=\chi^{\kappa}$, where $n=p^{\kappa}$. For $f$ of the form (1.1), the twisted Dirichlet series [5,6], is defined by

$$
f_{\chi}(s)=\sum_{n=1}^{+\infty} a_{n} \chi(n) n^{-s}
$$

Notice that if $\chi \in \mathbb{T}^{\infty}, f_{\chi}$ is the vertical limit of $f$, introduced in [19].

We also consider the translations $f_{\delta}(s)=f(s+\delta), \delta \in \mathbb{R}$. For those $\chi \in \mathbb{D}^{\infty}$ and $s=\sigma+i t$ for which the series (2.6) converges, we have

$$
f_{\chi}(s)=\left(\mathcal{B} f_{\sigma} \mathcal{T}_{t}\right)(\chi)
$$

When $f$ is in $\mathcal{H}_{w}^{2}$, the Cauchy-Schwarz inequality implies that (2.7) holds whenever $s \in \mathbb{C}_{1 / 2}$ and $\chi \in \overline{\mathbb{D}}^{\infty}$. By the Rademacher-Menchov Theorem (see [22]), (2.7) can be extended in the following way (the argument given in [5] for $\mathcal{B}_{\beta}^{2}$ remains true for $\left.\mathcal{A}_{\beta}^{2}\right)$.

Lemma 2 If $f$ is in $\mathcal{H}_{w}^{2}$, the Dirichlet series $f_{\chi}$ as defined in (2.6) converges in $\mathbb{C}_{0}$ for almost every $\chi \in \mathbb{D}^{\infty}$, with respect to $\mu_{w}$.

Recall that $\tau_{\theta}, \theta \in \mathbb{R}$, is the conformal mapping defined in (2.4). For $0<p<\infty$, the conformally invariant Hardy space $H_{i}^{p}\left(\mathbb{C}_{\theta}\right)$, is the space of those functions $f$ 
such that $f \circ \tau_{\theta}$ is in $H^{p}(\mathbb{T})$, the usual Hardy space of the unit disk. Setting $d \lambda(t)=$ $\pi^{-1}\left(1+t^{2}\right)^{-1} d t$, we get

$$
\|f\|_{H_{i}^{p}\left(\mathbb{C}_{\theta}\right)}^{p}=\int_{\mathbb{R}}|f(\theta+i t)|^{p} d \lambda(t)=\frac{1}{2 \pi} \int_{-\pi}^{\pi}\left|f \circ \tau_{\theta}(u)\right|^{p} d u, \text { for } f \in H_{i}^{p}\left(\mathbb{C}_{\theta}\right) .
$$

Let $f$ be in $\mathcal{H}_{w}^{p}$. In view of relation (2.3), and using the same argument as in [6,19], one can prove that for almost all $\chi$, with respect to $\mu_{w}, f_{\chi}$ can be extended analytically on $\mathbb{C}_{0}$ to an element of $H_{i}^{p}\left(\mathbb{C}_{0}\right)$. The norm of $f$ in $\mathcal{H}_{w}^{p}$ can be expressed as

$$
\|f\|_{\mathcal{H}_{w}^{p}}^{p}=\int_{\mathbb{D}^{\infty}}\left\|f_{\chi}\right\|_{H_{i}^{p}\left(\mathbb{C}_{0}\right)}^{p} d \mu_{w}(\chi)
$$

\subsection{A Littlewood-Paley formula}

We now derive another expression for the norm in $\mathcal{H}_{w}^{p}$.

Proposition 1 Let $\lambda$ be a probability measure on $\mathbb{R}$, and $p \geq 1$.

(a) If $f \in \mathcal{H}_{w}^{p}$, then $\|f\|_{\mathcal{H}_{w}^{p}}^{p} \asymp I_{p}(f)$, where

$$
\begin{aligned}
I_{p}(f):= & |f(+\infty)|^{p} \\
& +4 \int_{\mathbb{D}^{\infty}} \int_{\mathbb{R}} \int_{0}^{+\infty}\left|f_{\chi}(y+i t)\right|^{p-2}\left|f_{\chi}^{\prime}(y+i t)\right|^{2} y d y d \lambda(t) d \mu_{w}(\chi) .
\end{aligned}
$$

When $p=2$, we have $\|f\|_{\mathcal{H}_{w}^{2}}^{2}=I_{2}(f)$.

(b) Let $f \in \mathcal{D}, f(s)=\sum_{n=1}^{+\infty} a_{n} n^{-s}$, such that $f$ and $f_{\chi}$ converge on $\mathbb{C}_{0}$ for a.a. $\chi \in \mathbb{D}^{\infty}$. If $I_{p}(f)<\infty$, then $f \in \mathcal{H}_{w}^{p}$.

Proof Since the real variable $t$ corresponds to a rotation in each variable of $\mathbb{D}^{\infty}$, the rotation invariance of $\mu_{w}$ entails that $I_{p}(f)$ does not depend on the choice of the probability measure $\lambda$. For general $p \geq 1$, we prove (a), by using (2.8). We adapt the argument from [10] (for $\mathcal{H}^{p}$ ), by integrating over the polydisk $\mathbb{D}^{\infty}$ instead of the polytorus $\mathbb{T}^{\infty}$.

Suppose $f$ is in $\mathcal{H}_{w}^{2}$, and take $y>0$. From (2.3) and the rotation invariance, we obtain

$$
\begin{aligned}
\int_{\mathbb{R}} \int_{\mathbb{D} \infty}\left|f_{\chi}^{\prime}(y+i t)\right|^{2} d \mu_{w}(\chi) d \lambda(t) & =\int_{\mathbb{D} \infty}\left|\mathcal{B} f_{y}^{\prime}(\chi)\right|^{2} d \mu_{w}(\chi) \\
& =\sum_{n=1}^{+\infty} \frac{\left|a_{n}\right|^{2}}{w_{n}}(\log n)^{2} n^{-2 y}
\end{aligned}
$$

Integration against $y$ on $(0,+\infty)$ gives the formula (see details in [7] for the case of $\left.\mathcal{H}^{2}\right)$. 
If $f$ is as in (b), the integrand in $I_{p}(f)$ is measurable. For $\chi \in \mathbb{D}^{\infty}$, the change of variables $s=y+i t=\omega(z)=2 \frac{1+z}{1-z}$ transfers the Littlewood-Paley formula from $\mathbb{D}$ to $\mathbb{C}_{0}$,

$$
\begin{aligned}
\int_{\mathbb{R}} & \left|f_{\chi}(i t)\right|^{p} \frac{2}{\pi\left(2^{2}+t^{2}\right)} d t \\
\asymp & \left|f_{\chi}(2)\right|^{p} \\
& +\int_{\mathbb{D}}\left(1-|z|^{2}\right)\left|f_{\chi}(\omega(z))\right|^{p-2}\left|f_{\chi}^{\prime}(\omega(z))\right|^{2}\left|\omega^{\prime}(z)\right|^{2} d V(z) \\
\asymp & \left|f_{\chi}(2)\right|^{p} \\
& +\int_{0}^{+\infty} \int_{\mathbb{R}} \frac{2 y}{(y+2)^{2}+t^{2}}\left|f_{\chi}(y+i t)\right|^{p-2}\left|f_{\chi}^{\prime}(y+i t)\right|^{2} d t d y \\
\lesssim & \left\|f^{*}\right\|_{L^{\infty}\left(\overline{\mathbb{C}_{2}}\right)}^{p} \\
& +\int_{0}^{+\infty} \int_{\mathbb{R}} \frac{y}{1+t^{2}}\left|f_{\chi}(y+i t)\right|^{p-2}\left|f_{\chi}^{\prime}(y+i t)\right|^{2} d t d y,
\end{aligned}
$$

where $f^{*}(s):=\sum_{n=1}^{+\infty}\left|a_{n}\right| n^{-s}$ is bounded on $\overline{\mathbb{C}_{2}}$.

Integrating on $\mathbb{D}^{\infty}$ with respect to $\mu_{w}$, and using (2.3), we get that

$$
\|\mathcal{B} f\|_{L^{p}\left(\mathbb{D}^{\infty}, \mu_{w}\right)}^{p} \lesssim\left\|f^{*}\right\|_{L^{\infty}\left(\overline{\mathbb{C}_{2}}\right)}^{p}+I_{p}(f)<\infty
$$

Therefore, $\mathcal{B} f \in L^{p}\left(\mathbb{D}^{\infty}, \mu_{w}\right)$. The martingale $\left(A_{m} \mathcal{B} f\right)_{m}$ (with respect to the increasing sequence of $\sigma$-algebras of the sets $\left.\mathbb{D}^{m} \times\{0\}\right)$ converges in $L^{p}\left(\mathbb{D}^{\infty}, \mu_{w}\right)$ to $\mathcal{B} f$. Polynomial approximation in the Bergman spaces of the finite polydisks $\mathbb{D}^{m}$ shows that $\mathcal{B} f$ is in $\mathcal{B H}_{w}^{p}$.

\section{Spaces of symbols of Volterra operators in half-planes}

If $g$ is in $\mathcal{D}$, the definition (1.2) of $T_{g}$ shows that we can assume that $g(+\infty)=0$, i.e.

$$
g(s)=\sum_{n=2}^{+\infty} b_{n} n^{-s} .
$$

As in the study of Volterra operators on Bergman spaces the unit disk [2], and on the space of Dirichlet series $\mathcal{H}^{2}$ [13], the boundedness of $T_{g}$ on $\mathcal{H}_{w}^{2}$ will be related to Carleson measures, and to the membership of $g$ to a BMO space or a Bloch space.

Let $Y$ be either $\mathcal{H}_{w}^{2}$ or the Bergman space $A_{i, \delta}\left(\mathbb{C}_{1 / 2}\right), \delta>0$. A positive Borel measure $\mu$ on $\mathbb{C}_{1 / 2}$ is called a Carleson measure for $Y$ if there exists a constant $C$ such that, 


$$
\int_{\mathbb{C}_{1 / 2}}|f|^{2} d \mu \leq C\|f\|_{Y}^{2} \text { for all } f \in Y
$$

The smallest such constant, denoted by $\|\mu\|_{C M(Y)}$, is called the Carleson constant for $\mu$ with respect to $Y$. A Carleson measure $\mu$ is a vanishing Carleson measure for $Y$ if we have

$$
\lim _{k \rightarrow \infty} \int_{\mathbb{C}_{1 / 2}}\left|f_{k}\right|^{2} d \mu=0
$$

for every weakly compact sequence $\left(f_{k}\right)_{k}$ in $Y$ (which means that $\left\|f_{k}\right\|_{Y}$ is bounded and $f_{k}(s) \rightarrow 0$ on every compact set of $\left.\mathbb{C}_{1 / 2}\right)$.

\subsection{BMO spaces of Dirichlet series}

The space $B M O A\left(\mathbb{C}_{\theta}\right)$ consists of holomorphic functions $g$ in the half-plane $\mathbb{C}_{\theta}$ which satisfy

$$
\|g\|_{B M O\left(\mathbb{C}_{\theta}\right)}:=\sup _{I \subset \mathbb{R}} \frac{1}{|I|} \int_{I}\left|g(\theta+i t)-\frac{1}{|I|} \int_{I} g(\theta+i \tau) d \tau\right| d t<\infty .
$$

Any $g$ in $\mathcal{D} \cap B M O A\left(\mathbb{C}_{0}\right)$ has an abscissa of boundedness $\sigma_{b} \leq 0$ (Lemma 2.1 of [13]).

The space $\operatorname{VMOA}\left(\mathbb{C}_{0}\right)$ consists in those functions $g$ in $B M O A\left(\mathbb{C}_{0}\right)$ such that

$$
\lim _{\delta \rightarrow 0^{+}} \sup _{|I|<\delta} \frac{1}{|I|} \int_{I}\left|f(i t)-\frac{1}{|I|} \int_{I} f(i \tau) d \tau\right| d t=0 .
$$

\subsection{Bloch spaces of Dirichlet series}

The Bloch space Bloch $\left(\mathbb{C}_{\theta}\right)$ consists of holomorphic functions in the half-plane $\mathbb{C}_{\theta}$ which satisfy

$$
\|g\|_{\mathrm{Bloch}\left(\mathbb{C}_{\theta}\right)}:=\sup _{\sigma+i t \in \mathbb{C}_{\theta}}(\sigma-\theta)\left|f^{\prime}(\sigma+i t)\right| .
$$

Lemma 3 If $g$ be in $\mathcal{D} \cap \operatorname{Bloch}\left(\mathbb{C}_{0}\right)$.

(a) Its abscissa of boundedness satifies $\sigma_{b} \leq 0$.

(b) For every $\chi \in \mathbb{D}^{\infty}, g_{\chi}$ is in Bloch $\left(\mathbb{C}_{0}\right)$, and $\left\|g_{\chi}\right\|_{B l o c h}\left(\mathbb{C}_{0}\right) \leq\|g\|_{B \operatorname{Bloch}\left(\mathbb{C}_{0}\right)}$.

(c) Suppose that $y_{0}>\frac{1}{2}$. Then there exists a constant $C=C\left(y_{0}\right)$, such that,

$$
\left|g_{\chi}^{\prime}(y+i t)\right| \leq C 2^{-y}\|g\|_{B l o c h\left(\mathbb{C}_{0}\right)}, \text { for all } \chi \in \mathbb{D}^{\infty}, t \in \mathbb{R}, y \geq y_{0}
$$


Proof Let $\epsilon>0$. If $s=\sigma+i t$ is in $\mathbb{C}_{0}$, the definition of the Bloch-norm implies that

$$
\epsilon\left|g^{\prime}(\epsilon+s)\right| \leq(\epsilon+\sigma)\left|g^{\prime}(\epsilon+s)\right| \leq\|g\|_{\operatorname{Bloch}\left(\mathbb{C}_{0}\right)}
$$

It follows that $g^{\prime}$, and then $g$ is bounded in $\mathbb{C}_{\epsilon} ;$ (a) is proved.

Now fix $\sigma>0$. Let $m \geq 1$ be an integer, and $z=\left(z_{1}, \ldots, z_{m}, z_{m+1}, \ldots\right), \chi$ in $\mathbb{D}^{\infty}$. From the properties of $\mathcal{H}^{\infty}$ and the proof of (a), we have

$$
\left|A_{m} \mathcal{B}\left(g_{\sigma}^{\prime}\right)_{\chi}(z)\right|=\left|A_{m} \mathcal{B} g_{\sigma}^{\prime}(z \cdot \chi)\right| \leq\left\|\mathcal{B} g_{\sigma}^{\prime}\right\|_{H^{\infty}\left(\mathbb{T}^{\infty}\right)}=\left\|g_{\sigma}^{\prime}\right\|_{\mathcal{H}^{\infty}}
$$

and $\left\|\left(g_{\sigma}^{\prime}\right)_{\chi}\right\|_{\mathcal{H}^{\infty}}=\left\|\mathcal{B}\left(g_{\sigma}^{\prime}\right)_{\chi}\right\|_{H^{\infty}\left(\mathbb{T}^{\infty}\right)} \leq\left\|g_{\sigma}^{\prime}\right\|_{\mathcal{H}^{\infty}}$. Therefore, $\left(g_{\sigma}^{\prime}\right)_{\chi}$ is in $\mathcal{H}^{\infty} ;$ (b) holds, due to

$$
\sigma\left|g_{\chi}^{\prime}(\sigma+i t)\right| \leq\|g\|_{\operatorname{Bloch}\left(\mathbb{C}_{0}\right)}, \text { for all } t \in \mathbb{R}, \chi \in \mathbb{T}^{\infty}, \sigma>0 .
$$

If $0<\delta<y_{0}-\frac{1}{2}$, the Cauchy-Schwarz inequality and Parseval's relation induce that

$$
\begin{aligned}
\left|g_{\chi}^{\prime}(y+i t)\right|^{2} & \leq\left(\sum_{n=2}^{+\infty}\left|b_{n}\right|(\log n) n^{-y}\right)^{2}=\left(\sum_{n=2}^{+\infty}\left|b_{n}\right|(\log n) n^{-\frac{\delta}{2}} n^{-\left(\frac{\delta}{2}+\frac{1}{2}\right)} n^{-\left(y-\frac{1}{2}-\delta\right)}\right)^{2} \\
& \lesssim \zeta(1+\delta) 2^{-2 y}\left\|\mathcal{B} g_{\delta / 2}^{\prime}\right\|_{H^{2}\left(\mathbb{T}^{\infty}\right)}^{2}
\end{aligned}
$$

We now get (c) from the chain of inequalities

$$
\left\|\mathcal{B} g_{\delta / 2}^{\prime}\right\|_{H^{2}\left(\mathbb{T}^{\infty}\right)} \leq\left\|\mathcal{B} g_{\delta / 2}^{\prime}\right\|_{H^{\infty}\left(\mathbb{T}^{\infty}\right)}=\left\|g_{\delta / 2}^{\prime}\right\|_{\mathcal{H}^{\infty}} \leq \frac{2}{\delta}\|g\|_{\text {Bloch }\left(\mathbb{C}_{0}\right)},
$$

Now, recall several characterizations of Bloch functions, which are extracted from $[2,18]$.

Lemma 4 Assume $\delta>0$. For $g$ holomorphic in $\mathbb{C}_{\theta}$, the following are equivalent:

(a) $g \in \operatorname{Bloch}\left(\mathbb{C}_{\theta}\right)$;

(b) $h=g \circ \tau_{\theta} \in \operatorname{Bloch}(\mathbb{D})$;

(c) The measure $d \mu_{\mathbb{C}_{\theta}, g}(s)=\left|g^{\prime}(\sigma+i t)\right|^{2} \frac{(\sigma-\theta)^{\delta+1}}{|s-\theta+1|^{2 \delta+2}} d \sigma d t$ is a Carleson measure for $A_{i, \delta}\left(\mathbb{C}_{\theta}\right)$;

(d) The measure $d \mu_{\mathbb{D}, h}(z)=\left|h^{\prime}(z)\right|^{2}\left(1-|z|^{2}\right)^{\delta+1} d m_{1}(z)$ is a Carleson measure for $A_{\delta}^{2}(\mathbb{D})$;

(e) The operator $J_{h}$, given by

$$
J_{h} f(z)=\int_{0}^{z} f(t) h^{\prime}(t) d t
$$

is bounded on $A_{\delta}^{2}(\mathbb{D})$. 
Moreover, the quantities

$$
\|g\|_{\text {Bloch }\left(\mathbb{C}_{\theta}\right)},\left\|\mu_{\mathbb{C}_{\theta}, g}\right\|_{C M\left(\mathbb{C}_{\theta}\right)},\left\|J_{g}\right\|_{\mathcal{L}\left(A_{\delta}^{2}(\mathbb{D})\right)}
$$

are comparable.

The little Bloch space is the space

$$
\operatorname{Bloch}_{0}\left(\mathbb{C}_{\theta}\right)=\left\{f \in \operatorname{Bloch}\left(\mathbb{C}_{\theta}\right): \lim _{\sigma \rightarrow \theta}(\sigma-\theta)\left|g^{\prime}(s)\right|=0\right\} .
$$

The membership in $\operatorname{Bloch}_{0}\left(\mathbb{C}_{\theta}\right)$ is characterized by a little oh version of Lemma 4 , involving vanishing Carleson measures.

We show that Dirichlet polynomials are dense in $\mathcal{D} \cap \operatorname{Bloch}_{0}\left(\mathbb{C}_{0}\right)$. For $g(s)=$ $\sum_{n \geq 1} b_{n} n^{-s}$, the partial sum operator is defined by $S_{N} g(s)=\sum_{n=1}^{N} b_{n} n^{-s}$.

Proposition 2 Let $g$ be in Bloch $\left(\mathbb{C}_{0}\right) \cap \mathcal{D}$, and $\epsilon>0$. Then there exists $P$ in $\mathcal{P}$ such that

$$
\|g-P\|_{B l o c h\left(\mathbb{C}_{0}\right)} \leq \epsilon .
$$

If in addition $g$ is in $\mathcal{D}_{d}, P$ can be chosen in $\mathcal{D}_{d}$.

Proof For every $\delta>0, g_{\delta}=g(\delta+$.$) is also in Bloch \left(\mathbb{C}_{0}\right)$. As $\delta$ tends to $0,\left(g_{\delta}\right)_{\delta}$ converges to $g$ uniformly on compact sets of $\mathbb{C}_{0}$, and $\lim _{\sigma \rightarrow 0^{+}} \sigma\left|g_{\delta}^{\prime}(s)\right|=0$, uniformly with respect to $\delta \in(0,1)$. It then follows from [3] that $\lim _{\delta \rightarrow 0^{+}}\left\|g-g_{\delta}\right\|_{\operatorname{Bloch}\left(\mathbb{C}_{0}\right)}=$ 0 . Thus, we can choose $\delta>0$ such that $\left\|g-g_{\delta}\right\|_{\operatorname{Bloch}\left(\mathbb{C}_{0}\right)} \leq \frac{\epsilon}{2}$. Since $\sigma_{b}(g)=\sigma_{u}(g) \leq 0$, the partial sums $\left(S_{N} g\right)_{N}$ converge uniformly to $g$ in $\overline{\mathbb{C}_{\delta}}$, $\lim _{N \rightarrow+\infty}\left\|S_{N} g_{\delta}-g_{\delta}\right\|_{\mathcal{H}^{\infty}}=0$. For large $N$, the triangle inequality implies that

$$
\begin{aligned}
\left\|g-S_{N} g_{\delta}\right\|_{\mathrm{Bloch}\left(\mathbb{C}_{0}\right)} & \leq\left\|g-g_{\delta}\right\|_{\mathrm{Bloch}\left(\mathbb{C}_{0}\right)}+\left\|g_{\delta}-S_{N} g_{\delta}\right\|_{\mathrm{Bloch}\left(\mathbb{C}_{0}\right)} \\
& \leq \frac{\epsilon}{2}+2\left\|S_{N} g_{\delta}-g_{\delta}\right\|_{\mathcal{H}^{\infty}} \leq \epsilon
\end{aligned}
$$

\subsection{Carleson measures on the half-plane $\mathbb{C}_{1 / 2}$}

On $\mathbb{C}_{1 / 2}$, we consider Carleson squares

$$
Q\left(s_{0}\right)=\left(\frac{1}{2}, \sigma_{0}\right] \times\left[t_{0}-\frac{\epsilon}{2}, t_{0}+\frac{\epsilon}{2}\right], \text { where } s_{0}=\sigma_{0}+i t_{0} \in \mathbb{C}_{1 / 2}
$$

is the midpoint of the right edge of the square and $\epsilon=\sigma_{0}-\frac{1}{2}$.

We need the following property (see Section 7.2 in [31]). 
Lemma 5 Let $\delta>0$ and let $\mu$ be a Borel measure on $\mathbb{C}_{1 / 2}$. Then $\mu$ is a Carleson measure for $A_{i, \delta}\left(\mathbb{C}_{1 / 2}\right)$ if and only if, for every square $Q\left(s_{0}\right)$, with $s_{0}=\sigma_{0}+i t_{0}$, we have

$$
\mu\left(Q\left(s_{0}\right)\right)=O\left(\left(2 \sigma_{0}-1\right)^{\delta+1}\right) \text { as } \sigma_{0} \rightarrow\left(\frac{1}{2}\right)^{+}
$$

In addition, $\mu$ is a vanishing Carleson measure for $A_{i, \delta}\left(\mathbb{C}_{1 / 2}\right)$ if and only if, uniformly for $t_{0}$ in $\mathbb{R}$,

$$
\mu\left(Q\left(s_{0}\right)\right)=o\left(\left(2 \sigma_{0}-1\right)^{\delta+1}\right) \text { as } \sigma_{0} \rightarrow\left(\frac{1}{2}\right)^{+}
$$

By Lemma $1, \mathcal{H}_{w}^{2}$ is embedded in the Bergman-type space $A_{i, \delta}\left(\mathbb{C}_{1 / 2}\right)$, the exponent $\delta=\delta(w)$ being defined in (2.5). Bounded Carleson measures for both spaces $\mathcal{H}_{w}^{2}$ and $A_{i, \delta}\left(\mathbb{C}_{1 / 2}\right)$ have been compared in $[8,23,24]$. We extend their results.

Lemma 6 Let $\mu$ be a positive Borel measure on $\mathbb{C}_{1 / 2}$.

(1) If $\mu$ is a Carleson measure (resp. vanishing Carleson measure) for $\mathcal{H}_{w}^{2}$, then $\mu$ is a Carleson measure (resp. vanishing Carleson measure) for $A_{i, \delta}\left(\mathbb{C}_{1 / 2}\right)$ and

$$
\|\mu\|_{C M\left(A_{i, \delta}\left(\mathbb{C}_{1 / 2}\right)\right)} \lesssim\|\mu\|_{C M\left(\mathcal{H}_{w}^{2}\right)}
$$

(2) Assume that $\mu$ has bounded support. If $\mu$ is a Carleson measure (resp. vanishing Carleson measure) for $A_{i, \delta}\left(\mathbb{C}_{1 / 2}\right)$, then $\mu$ is a Carleson measure (resp. vanishing Carleson measure) for $\mathcal{H}_{w}^{2}$ and

$$
\|\mu\|_{C M\left(\mathcal{H}_{w}^{2}\right)} \lesssim\|\mu\|_{C M\left(A_{i, \delta}\left(\mathbb{C}_{1 / 2}\right)\right)}
$$

Proof Suppose that $\mu$ is a Carleson measure for $\mathcal{H}_{w}^{2}$, and let $Q\left(s_{0}\right)$ be a small Carleson square in $\mathbb{C}_{1 / 2}$. For the test function $f_{s_{0}}(s)=K^{\mathcal{H}_{w}^{2}}\left(s, s_{0}\right)$, we have

$$
\int_{Q\left(s_{0}\right)}\left|f_{s_{0}}\right|^{2} d \mu \leq \int_{\mathbb{C}_{1 / 2}}\left|f_{s_{0}}\right|^{2} d \mu \leq C(\mu)\left\|K^{\mathcal{H}_{w}^{2}}\left(., s_{0}\right)\right\|_{\mathcal{H}_{w}^{2}}^{2} \lesssim Z_{w}\left(\Re s_{0}\right)
$$

From the estimate of $Z_{w}(2.1)$ and Lemma $5, \mu$ is a Carleson measure for $A_{i, \delta}\left(\mathbb{C}_{1 / 2}\right)$, since

$$
\left(\Re s_{0}-\frac{1}{2}\right)^{-2(\delta+1)} \mu\left(Q\left(s_{0}\right)\right) \lesssim\left(\Re s_{0}-\frac{1}{2}\right)^{-(\delta+1)}
$$

For $\mu$ a Carleson measure for $A_{i, \delta}\left(\mathbb{C}_{1 / 2}\right)$ with bounded support, (2) holds [23,24]. 
As for vanishing Carleson measures, the reasoning used in [8] for $\mathcal{B}_{\beta}^{2}$ can be transfered to the spaces $\mathcal{A}_{\beta}^{2}$, with the test functions

$$
f_{k}(s)=\frac{K^{\mathcal{H}_{w}^{2}}\left(s, s_{k}\right)}{\left\|K^{\mathcal{H}_{w}^{2}}\left(., s_{k}\right)\right\|_{\mathcal{H}_{w}^{2}}}
$$

where $s_{k}=1 / 2+\epsilon_{k}+i \tau_{k}$ is a sequence in $\mathbb{C}_{1 / 2}$ such that $\epsilon_{k} \rightarrow 0$.

We also require an equivalent norm for $A_{i, \delta}\left(\mathbb{C}_{1 / 2}\right)$, when $\delta>0$. For Bergman spaces of the unit disk, recall the following consequence of Stanton's formula [28,29]:

$$
\|h\|_{A_{\delta}(\mathbb{D})}^{2} \asymp|h(0)|^{2}+\int_{\mathbb{D}}\left|h^{\prime}(z)\right|^{2}\left(1-|z|^{2}\right)^{\delta+1} d V(z), \text { for } h \text { holomorphic on } \mathbb{D} \text {. }
$$

Via the mapping $\tau_{1 / 2}$, we obtain that, for any $f$ holomorphic on $\mathbb{C}_{1 / 2}$,

$$
\|f\|_{A_{i, \delta}\left(\mathbb{C}_{1 / 2}\right)}^{2} \asymp\left|f\left(\frac{3}{2}\right)\right|^{2}+\int_{\mathbb{C}_{1 / 2}}\left|f^{\prime}(s)\right|^{2} \frac{\left(\sigma-\frac{1}{2}\right)^{\delta+1}}{\left|s+\frac{1}{2}\right|^{2 \delta+2}} d V(s) .
$$

\section{Boundedness of $T_{g}$}

In this section, we characterize functions in $\mathcal{X}_{w}$, and prove Theorem 1.

\subsection{Carleson measure characterization}

The boundedness of $T_{g}$ on $\mathcal{H}_{w}^{2}$ can be described in terms of Carleson measures. This generalizes the setting of the Hardy space $\mathcal{H}^{2}$ [13].

Recall that $\mathcal{H}_{w}^{2}$ is associated to the probability measure $\mu_{w}$ on the polydisk $\mathbb{D}^{\infty}$.

Proposition $3 T_{g}$ is bounded on $\mathcal{H}_{w}^{2}$ if and only if there exists a constant $C=C(\mathrm{~g})$ such that

$$
\begin{aligned}
\left\|T_{g} f\right\|_{\mathcal{H}_{w}^{2}}^{2} & \asymp \int_{\mathbb{D} \infty} \int_{\mathbb{R}} \int_{0}^{+\infty}\left|f_{\chi}(\sigma+i t)\right|^{2}\left|g_{\chi}^{\prime}(\sigma+i t)\right|^{2} \frac{\sigma d \sigma d t}{1+t^{2}} d \mu_{w}(\chi) \\
& \leq C^{2}\|f\|_{\mathcal{H}_{w}^{2}}^{2},
\end{aligned}
$$

or, equivalently

$$
\int_{\mathbb{D}^{\infty}} \int_{0}^{+\infty}\left|f_{\chi}(\sigma)\right|^{2}\left|g_{\chi}^{\prime}(\sigma)\right|^{2} \sigma d \sigma d \mu_{w}(\chi) \leq C^{2}\|f\|_{\mathcal{H}_{w}^{2}}^{2} .
$$

The smallest constant $C$ satisfying (4.1) is such that $C \asymp\left\|T_{g}\right\|_{\mathcal{L}\left(\mathcal{H}_{w}^{2}\right)}$. 
Proof Applying the Littlewood-Paley formula (Proposition 1) to the measure $d \lambda(t)=$ $\pi^{-1}\left(1+t^{2}\right)^{-1} d t$ and the function $T_{g} f$, we get (4.1).

The rotation invariance of the measure $d \mu_{w}(\chi)$ gives (4.2).

\subsection{Proof of Theorem 1 (a): $\mathcal{B g}$ depends on a finite number of variables}

For $1 \leq q$ and $d \geq 1$, recall that $f \in \mathcal{H}_{d, w}^{q}$ if and only if $f$ is in $\mathcal{H}_{w}^{q}$ and $\mathcal{B} f$ is a function of $z_{1}, \ldots, z_{d}$.

When needed, we shall identify $z=\left(z_{1}, \ldots, z_{d}\right) \in \mathbb{D}^{d}$ with $(z, 0) \in \mathbb{D}^{d} \times\{0\}$.

If $g(s)=\sum_{n=2}^{+\infty} b_{n} n^{-s}$ is in $\mathcal{H}_{d, w}^{2}$, we observe that for $z \in \mathbb{D}^{d}$,

$$
\mathcal{B} g^{\prime}(z)=\sum_{j=1}^{d} \log p_{j} \sum_{\alpha \in \mathbb{N}^{d}} \tilde{b}_{\alpha} \alpha_{j} z^{\alpha}=\operatorname{RBg}(z)
$$

where $R$ is the operator

$$
R G\left(z_{1}, \ldots, z_{d}\right)=\sum_{j=1}^{d}\left(\log p_{j}\right) z_{j} \partial_{j} G\left(z_{1}, \ldots, z_{d}\right)
$$

We define the set

$$
\Delta_{\epsilon}:=\left\{z=\left(z_{1}, \ldots, z_{d}\right) \in \mathbb{D}^{d}, \forall j,\left|z_{j}\right|<p_{j}^{-\epsilon}\right\}, \text { for } \epsilon>0
$$

Take $x>0, t \in \mathbb{R}$, and $z \in \mathbb{D}^{d}$. By construction, $z \in \overline{\Delta_{\sigma(z)}}$ and $\sigma\left(\mathfrak{p}^{-\mathbf{x}} . z\right) \geq$ $\sigma(z)+x \frac{\log p_{1}}{\log p_{d}}$.

For $g \in \mathcal{D}_{d}$, we write $g_{z}(x)=g_{(z, 0)}(x)=\mathcal{B} g_{x}(z)$. Since $g$ is in $\operatorname{Bloch}\left(\mathbb{C}_{0}\right)$, we apply (1.6) to $g_{x}^{\prime}$, and get

$$
\begin{aligned}
& \left|g_{z}^{\prime}(x+i t)\right|=\left|\mathcal{B} g_{x}^{\prime}\left(\mathcal{T}_{t} z\right)\right| \leq \sup _{\zeta \in \overline{\Delta_{\sigma\left(\mathfrak{p}-\mathbf{x}_{. z)}\right.}}}\left|\mathcal{B} g^{\prime}(\zeta)\right| \\
& =\sup _{s \in \overline{\mathbb{C}}_{\sigma\left(\mathfrak{p}^{-\mathbf{x}_{. z)}}\right.}}\left|g^{\prime}(s)\right| \leq \frac{\log p_{d}}{\log p_{1}} \frac{\|g\|_{\mathrm{Bloch}\left(\mathbb{C}_{0}\right)}}{x+\sigma(z)},
\end{aligned}
$$

Proof of Theorem 1(a) Let $f(s)=\sum_{n \geq 1} a_{n} n^{-s}$ be in $\mathcal{H}_{w}^{2}$, and, for $\chi=\left(z, z^{\prime}\right) \in$ $\mathbb{D}^{d} \times \mathbb{D}^{\infty}$,

$\mathcal{B} f(\chi)=\sum_{\left(\alpha, \alpha^{\prime}\right) \in \mathbb{N}^{d} \times \mathbb{N}_{0, \mathrm{fin}}^{\infty}} c_{\alpha, \alpha^{\prime}} z^{\alpha} z^{\prime \alpha^{\prime}}=\sum_{\alpha \in \mathbb{N}^{d}} c_{\alpha}^{\prime}\left(z^{\prime}\right) z^{\alpha}$, where $c_{\alpha}^{\prime}\left(z^{\prime}\right)=\sum_{\alpha^{\prime} \in \mathbb{N}_{0, \text { fin }}^{\infty}} c_{\alpha, \alpha^{\prime}} z^{\alpha^{\prime}}$ 
In view of Proposition 3, we aim to estimate $\left\|T_{g} f\right\|_{\mathcal{H}_{w}^{2}}^{2} \asymp \mathcal{I}_{1}+\mathcal{I}_{2}$, where

$$
\begin{aligned}
\mathcal{I}_{1} & :=\int_{\mathbb{D}^{\infty}} \int_{0}^{1}\left|f_{\chi}(x)\right|^{2}\left|g_{\chi}^{\prime}(x)\right|^{2} x d x d \mu_{w}(\chi), \\
\text { and } \mathcal{I}_{2} & :=\int_{\mathbb{D}^{\infty}} \int_{1}^{+\infty}\left|f_{\chi}(x)\right|^{2}\left|g_{\chi}^{\prime}(x)\right|^{2} x d x d \mu_{w}(\chi) .
\end{aligned}
$$

By (4.3), the rotation invariance and Fubini's Theorem, we have

$$
\begin{aligned}
\mathcal{I}_{1} & \lesssim\|g\|_{\mathrm{Bloch}\left(\mathbb{C}_{0}\right)}^{2} \int_{0}^{1} x \int_{\mathbb{D}^{\infty}} \int_{\mathbb{D}^{d}} \frac{1}{[x+\sigma(z)]^{2}} \\
& \left|\sum_{\alpha \in \mathbb{N}^{d}} c_{\alpha}^{\prime}\left(\mathfrak{p}^{\prime-\mathbf{x}} \cdot z^{\prime}\right)\left(z_{1} p_{1}^{-x}\right)^{\alpha_{1}} \cdots\left(z_{d} p_{d}^{-x}\right)^{\alpha_{d}}\right|^{2} d \mu_{w}\left(z, z^{\prime}\right) d x \\
& \lesssim\|g\|_{\mathrm{Bloch}\left(\mathbb{C}_{0}\right)}^{2} \int_{\mathbb{D}^{\infty}} \int_{0}^{1} x \sum_{\alpha \in \mathbb{N}^{d}}\left|c_{\alpha}^{\prime}\left(\mathfrak{p}^{\prime-\mathbf{x}} \cdot z^{\prime}\right)\right|^{2} I_{\alpha}(x) d x d \mu_{w}\left(z^{\prime}\right),
\end{aligned}
$$

where

$$
I_{\alpha}(x):=\int_{\mathbb{D}^{d}} \frac{1}{[x+\sigma(z)]^{2}}\left|z_{1} p_{1}^{-x}\right|^{2 \alpha_{1}} \cdots\left|z_{d} p_{d}^{-x}\right|^{2 \alpha_{d}} d \mu_{w}(z)
$$

Using the rotation invariance again as well as the fact that $p_{j} \geq 1$, and setting $\mathcal{J}_{\alpha}:=$ $\int_{0}^{1} x I_{\alpha}(x) d x$, we get

$$
\begin{aligned}
\mathcal{I}_{1} & \lesssim\|g\|_{\mathrm{Bloch}\left(\mathbb{C}_{0}\right)}^{2} \sum_{\alpha \in \mathbb{N}^{d}} \int_{0}^{1} x I_{\alpha}(x)\left(\int_{\mathbb{D} \infty}\left|\sum_{\alpha^{\prime}} c_{\alpha, \alpha^{\prime}}\left(\mathfrak{p}^{\prime-\mathbf{x}} \cdot z^{\prime}\right)^{\alpha^{\prime}}\right|^{2} d \mu_{w}\left(z^{\prime}\right)\right) d x \\
& \lesssim\|g\|_{\mathrm{Bloch}\left(\mathbb{C}_{0}\right)}^{2} \sum_{\alpha, \alpha^{\prime}}\left|c_{\alpha, \alpha^{\prime}}\right|^{2} \mathcal{J}_{\alpha}\left(\int_{\mathbb{D} \infty}\left|z^{\prime \alpha^{\prime}}\right|^{2} d \mu_{w}\left(z^{\prime}\right)\right) \\
& \lesssim\|g\|_{\mathrm{Bloch}\left(\mathbb{C}_{0}\right)}^{2} \sum_{\alpha, \alpha^{\prime}} \frac{\left|c_{\alpha, \alpha^{\prime}}\right|^{2} \mathcal{J}_{\alpha}}{w\left(p_{d+1}^{\alpha_{d+1}}\right) \cdots w\left(p_{r}^{\alpha_{r}}\right)} .
\end{aligned}
$$

For the moment, we admit that $\mathcal{J}_{\alpha} \leq C(d, w)\left[\prod_{j=1}^{d} w\left(p_{j}^{\alpha_{j}}\right)\right]^{-1}$, which will be proved in Lemma 7. Hence,

$$
\mathcal{I}_{1} \lesssim\|g\|_{\mathrm{Bloch}\left(\mathbb{C}_{0}\right)}^{2} \sum_{\alpha, \alpha^{\prime}} \frac{\left|c_{\alpha, \alpha^{\prime}}\right|^{2}}{w\left(p^{\left(\alpha, \alpha^{\prime}\right)}\right)} \lesssim\|g\|_{\mathrm{Bloch}\left(\mathbb{C}_{0}\right)}^{2}\|f\|_{\mathcal{H}_{w}^{2}}^{2} .
$$


Combining Lemma 3 with the following observation,

$$
\begin{aligned}
\int_{\mathbb{D}^{\infty}}\left|f_{\chi}(x)\right|^{2} d \mu_{w}(\chi) & =\int_{\mathbb{D}^{\infty}}\left|\sum_{n=p^{\alpha}} a_{n} n^{-x} \chi^{\alpha}\right|^{2} d \mu_{w}(\chi) \\
& =\sum_{n \geq 1} \frac{\left|a_{n}\right|^{2} n^{-2 x}}{w_{n}} \leq\|f\|_{\mathcal{H}_{w}^{2}}^{2},
\end{aligned}
$$

we estimate $\mathcal{I}_{2}$,

$\mathcal{I}_{2} \lesssim \int_{1}^{+\infty} x \int_{\mathbb{D} \infty}\|g\|_{\operatorname{Bloch}\left(\mathbb{C}_{0}\right)}^{2} 4^{-x}\left|f_{\chi}(x)\right|^{2} d \mu_{w}(\chi) d x \lesssim\|g\|^{2} \mid \operatorname{Bloch}\left(\mathbb{C}_{0}\right)\|f\|_{\mathcal{H}_{w}^{2}}^{2}$

Recall that

$$
I_{\alpha}(x)=\int_{\mathbb{D}^{d}} \frac{1}{[x+\sigma(z)]^{2}}\left|z_{1} p_{1}^{-x}\right|^{2 \alpha_{1}} \cdots\left|z_{d} p_{d}^{-x}\right|^{2 \alpha_{d}} d \mu_{w}(z), \alpha \in \mathbb{N}^{d}, 0<x<1 .
$$

Lemma 7 There exists a constant $C=C(w, d)$, such that

$$
\mathcal{J}_{\alpha}:=\int_{0}^{1} x I_{\alpha}(x) d x \leq C \prod_{j=1}^{d} \frac{1}{w\left(p_{j}^{\alpha_{j}}\right)}
$$

The proof of Lemma 7 relies on technical computations (Lemma 8).

Lemma 8 For $0<T<1$, and a real number $p \geq 2$, set $L:=-\frac{\log T}{2 \log p}$ and $K=$ $\min (1, L)$. There exists a constant $C=C(p, w)>0$, such that

$$
\begin{aligned}
J(p, T) & :=(\log T)^{-2} \int_{0}^{K} x M\left(T p^{2 x}\right) d x \\
& \lesssim C \begin{cases}M(T) & \text { if } \beta \geq 1 \text { or }\left(\beta<1, p^{-2}<T<1\right) \\
M\left(T p^{2}\right) & \text { if } \beta<1,0<T \leq p^{-2}\end{cases}
\end{aligned}
$$

Proof When $p^{-2}<T<1$, the change of variables $u=T p^{2 x}$ gives

$$
J(p, T)=(\log T)^{-2} \frac{1}{(2 \log p)^{2}} \int_{T}^{1} \log \frac{u}{T} M(u) \frac{d u}{u} .
$$

Since $\log \frac{u}{T} \leq \log \frac{1}{T}$ and $1 \leq \frac{1}{u} \leq \frac{1}{T}<p^{2}$,

$$
J(p, T) \leq(\log T)^{-2}\left(\frac{1}{2 \log p}\right)^{2} \int_{T}^{1} \log \frac{1}{T} M(u) \frac{1}{u} d u \lesssim M(T) .
$$


Next suppose that $0<T \leq p^{-2}$. Since $(\log T)^{2} \geq 4(\log p)^{2}$, we notice that

$$
J(p, T) \lesssim \int_{0}^{1} x M\left(T p^{2 x}\right) d x \lesssim\left\{\begin{array}{l}
\int_{0}^{1} M(T) d x \text { if } \beta \geq 1 \\
\int_{0}^{1} M\left(T p^{2}\right) d x \text { if } \beta<1
\end{array} .\right.
$$

Proof of Lemma 7 Resorting to polar coordinates, and using changes of variables, we have

$$
\mathcal{J}_{\alpha} \leq \int_{Q} \frac{x t^{\alpha}}{\left[x+\sigma\left(p_{1}^{x} \sqrt{t_{1}}, \ldots, p_{1}^{x} \sqrt{t_{d}}\right)\right]^{2}}\left(\prod_{k=1}^{d} M\left(p_{k}^{2 x} t_{k}\right) p_{k}^{2 x}\right) d x d t_{1} \cdots d t_{d}
$$

where $Q=\left\{(x, t) \in(0,1) \times(0,1)^{d}, \forall k=1 . . d, 0<t_{k}<p_{k}^{-2 x}\right\}$.

For $t=\left(t_{1}, \ldots, t_{d}\right) \in(0,1)^{d}$, set

$$
\begin{aligned}
l_{k}(t) & :=-\frac{\log t_{k}}{2 \log p_{k}}, K_{k}:=\min \left(1, l_{k}\right), 1 \leq k \leq d, \\
l(t) & :=\min _{1 \leq k \leq d} l_{k}(t), \quad K:=\min (1, l) .
\end{aligned}
$$

We observe that $Q=\left\{(x, t) \in(0,1) \times(0,1)^{d}, 0<x<K(t)\right\}$. Now, for $1 \leq$ $k \leq d$, we set $Q_{k}:=\left\{(x, t), t \in(0,1)^{d}, l(t)=l_{k}(t), 0<x<K_{k}(t)\right\}$.

Let $(x, t)$ be in $Q_{k}$. We have

$$
0<t_{l} \leq T_{k, l}:=t_{k}^{\frac{\log p_{l}}{\log p_{k}}}<1, \text { for } 1 \leq l \leq d .
$$

In addition, since $0<x<l_{k}(t)$, (4.4) implies $p_{l}^{x} \sqrt{t_{l}}<p_{l}^{l_{k}(t)} \sqrt{t_{l}} \leq 1$, and we see that $\frac{1}{\sqrt{t_{l}} p_{l}^{x}} \geq p_{l}^{l_{k}(t)-x} \geq p_{1}^{l_{k}(t)-x}$. Thus

$$
\left(\log p_{d}\right) \sigma\left(p_{1}^{x} \sqrt{t_{1}}, \ldots, p_{d}^{x} \sqrt{t_{d}}\right)=\log \min _{1 \leq l \leq d}\left(\frac{1}{\sqrt{t_{l}} p_{l}^{x}}\right) \geq \log p_{1}\left(l_{k}(t)-x\right),
$$

and $x+\sigma\left(p_{1}^{x} \sqrt{t_{1}}, \ldots, p_{1}^{x} \sqrt{t_{d}}\right) \gtrsim-\log t_{k}$.

Set $d \widehat{t_{k}}=d t_{1} \cdots d t_{k-1} d t_{k+1} \cdots d t_{d}$, and

$$
\tilde{Q}_{k}:=\left\{(x, t), 0<t_{k}<1,0<t_{l}<T_{k, l} \text { for } l \neq k, 0<x<K_{k}(t)\right\} .
$$

It follows that $\mathcal{J}_{\alpha} \lesssim \sum_{k=1}^{d} \mathcal{J}_{\alpha, k}$, where

$$
\mathcal{J}_{\alpha, k}=\int_{\tilde{Q}_{k}} \frac{x t^{\alpha}}{\left[x+\sigma\left(p_{1}^{x} \sqrt{t_{1}}, \ldots, p_{1}^{x} \sqrt{t_{d}}\right)\right]^{2}}\left(\prod_{l=1}^{d} M\left(p_{l}^{2 x} t_{l}\right)\right) d x d t
$$


We will obtain the Lemma by showing that

$$
\mathcal{J}_{\alpha, k} \lesssim \prod_{l=1}^{d}\left[w\left(p_{l}^{\alpha_{l}}\right)\right]^{-1}
$$

When $\beta \geq 1$, we use that, for $(x, t) \in \tilde{Q}_{k}$, and $l \neq k, M\left(p_{l}^{2 x} t_{l}\right) \leq M\left(t_{l}\right)$, altogether with Lemma 8. We derive (4.5) from

$$
\begin{aligned}
\mathcal{J}_{\alpha, k} & \lesssim \int_{0<t_{k}<1}\left(\int_{\prod_{j \neq k}\left(0, T_{k, j}\right)} t^{\alpha} \int_{0}^{K_{k}(t)} x\left(\log t_{k}\right)^{-2} M\left(p_{k}^{2 x} t_{k}\right) d x \prod_{l \neq k} M\left(t_{l}\right) d \widehat{t_{k}}\right) d t_{k} \\
& \lesssim \int_{0<t_{k}<1} t_{k}^{\alpha_{k}} M\left(t_{k}\right)\left(\prod_{j \neq k} \int_{0}^{T_{k, j}} t_{j}^{\alpha_{j}} M\left(t_{j}\right) d t_{j}\right) d t_{k} \lesssim \prod_{j=1}^{d} \int_{0}^{1} t_{j}^{\alpha_{j}} M\left(t_{j}\right) d t_{j} .
\end{aligned}
$$

Next, suppose $0<\beta<1$. If $(x, t) \in \tilde{Q}_{k}$, notice that, for $l \neq k, t_{l} p_{l}^{2 x} \leq t_{l} p_{l}^{2 l_{k}(t)} \leq$ 1 ; this shows that $M\left(p_{l}^{2 x} t_{l}\right) \leq M\left(p_{l}^{2 l_{k}(t)} t_{l}\right)$. Hence, we see that $\mathcal{J}_{\alpha, k} \lesssim J_{1}+J_{2}$, where, by Lemma 8 and the relation $p_{l}^{2 l_{k}(t)}=T_{k, l}^{-1}$,

$$
\begin{aligned}
& J_{1} \lesssim \int_{0<t_{k}<p_{k}^{-2}} t_{k}^{\alpha_{k}} M\left(p_{k}^{2} t_{k}\right)\left(\prod_{j \neq k} \int_{0}^{T_{k, j}} t_{j}^{\alpha_{j}} M\left(t_{j} T_{k, j}^{-1}\right) d t_{j}\right) d t_{k}, \\
& J_{2} \lesssim \int_{p_{k}^{-2}<t_{k}<1} t_{k}^{\alpha_{k}} M\left(t_{k}\right)\left(\prod_{j \neq k} \int_{0}^{T_{k, j}} t_{j}^{\alpha_{j}} M\left(t_{j} T_{k, j}^{-1}\right) d t_{j}\right) d t_{k} .
\end{aligned}
$$

A change of variables provides the desired estimate.

\subsection{Proof of Theorem 1(b) and (c)}

If $f(s)=\sum_{n=1}^{+\infty} a_{n} n^{-s}$ and $g(s)=\sum_{n=1}^{+\infty} b_{n} n^{-s}$, we have

$$
T_{g} f(s)=\sum_{n=2}^{\infty} \frac{1}{\log n}\left(\sum_{k \mid n, k<n} a_{k} b_{n / k}\right) n^{-s} .
$$

As in the case of $\mathcal{H}^{2}$, the operator

$$
a_{1}+\sum_{n=2}^{\infty} a_{n} n^{-s} \mapsto a_{1}+\sum_{n=2}^{\infty} a_{n}(\log n)^{-1} n^{-s}
$$


is compact on $\mathcal{H}_{w}$. Thus, set $b_{1}=1$, and our study will be unchanged if we replace $T_{g}$ by

$$
\tilde{T}_{g} f(s)=\sum_{n=2}^{\infty} \frac{1}{\log n}\left(\sum_{k \mid n} a_{k} b_{n / k}\right) n^{-s}
$$

Lemma 9 If $T_{g}$ is bounded on $\mathcal{H}^{2}$, then $g$ is in $\mathcal{X}_{w}$, and the operator norms satisfy

$$
\left\|T_{g}\right\|_{\mathcal{L}\left(\mathcal{H}_{w}^{2}\right)} \leq\left\|T_{g}\right\|_{\mathcal{L}\left(\mathcal{H}^{2}\right)} .
$$

Proof If $f(s)=\sum_{n=1}^{+\infty} a_{n} n^{-s}$ is in $\mathcal{H}_{w}^{2}$, the function $\tilde{f}(s)=\sum_{n=1}^{+\infty} a_{n} w_{n}^{-1 / 2} n^{-s}$ is in $\mathcal{H}^{2}$ and $\|f\|_{\mathcal{H}_{w}^{2}}=\|\tilde{f}\|_{\mathcal{H}^{2}}$. Since $w_{k} \leq w_{k l}$ for any integers $k, l$, the Lemma is proven by the inequality

$$
\left\|T_{g} f\right\|_{\mathcal{H}_{w}^{2}}^{2} \leq \sum_{n=2}^{\infty}(\log n)^{-2}\left|\sum_{k \mid n, k<n} w_{k}^{-1 / 2} a_{k} b_{n / k}\right|^{2}=\left\|T_{g} \tilde{f}\right\|_{\mathcal{H}^{2}}^{2} .
$$

We will also use the sufficient condition proved in Theorem 2.3 in [13], stating that if $g$ is in $B M O A\left(\mathbb{C}_{0}\right) \cap \mathcal{D}$, then $T_{g}$ is bounded on $\mathcal{H}^{2}$, with

$$
\left\|T_{g}\right\|_{\mathcal{H}^{2}} \lesssim\|g\|_{B M O A\left(\mathbb{C}_{0}\right)}
$$

Proof of Theorem 1(b) and (c) If $g$ is in $B M O A\left(\mathbb{C}_{0}\right), T_{g}$ is bounded on $\mathcal{H}^{2}$, and (b) is a consequence of (4.6) and Lemma 9.

To prove (c), we use that $\left(T_{g} f\right)^{\prime}=f g^{\prime}$, and that $\mathcal{H}_{w}^{2}$ is embedded in $A_{i, \delta}\left(\mathbb{C}_{1 / 2}\right)$, with $\delta=\delta(w)>0$. We set

$$
d v_{g}(s)=\left|g^{\prime}(s)\right|^{2} \frac{\left(\sigma-\frac{1}{2}\right)^{\delta+1}}{\left|s+\frac{1}{2}\right|^{2(\delta+1)}} d V(s)
$$

Now formula (3.1), the boundedness of $T_{g}$ on $\mathcal{H}_{w}^{2}$ and Lemma 1 induce that

$$
\int_{\mathbb{C}_{1 / 2}}|f(s)|^{2} d \nu_{g}(s) \lesssim\left\|T_{g} f\right\|_{A_{i, \delta}\left(\mathbb{C}_{1 / 2}\right)}^{2} \leq c(w)\left\|T_{g} f\right\|_{\mathcal{H}_{w}^{2}}^{2} \leq c(w)\left\|T_{g}\right\|_{\mathcal{L}\left(\mathcal{H}_{w}^{2}\right)}^{2}\|f\|_{\mathcal{H}_{w}^{2}}^{2},
$$

Thus, $v_{g}$ is a Carleson measure for $\mathcal{H}_{w}^{2}$ and $\left\|v_{g}\right\|_{C M\left(\mathcal{H}_{w}^{2}\right)} \lesssim\left\|T_{g}\right\|_{\mathcal{L}\left(\mathcal{H}_{w}^{2}\right)}^{2}$. By Lemma 6, $\nu_{g}$ is also a Carleson measure for $A_{i, \delta}\left(\mathbb{C}_{1 / 2}\right)$ and

$$
\left\|v_{g}\right\|_{C M\left(A_{i, \delta}\left(\mathbb{C}_{1 / 2}\right)\right)} \lesssim\left\|T_{g}\right\|_{\mathcal{L}\left(\mathcal{H}_{w}^{2}\right)}^{2}
$$


We conclude by the characterization of the Bloch space given in Lemma 4.

We get a result which is in agreement with the situation for Hardy spaces [15], Bergman spaces [2] or the Hardy space of Dirichlet series $\mathcal{H}^{2}$ [13], with the same proof.

Corollary 1 If $g$ is in $\mathcal{X}_{w}$, then $g$ is in $\cap_{0<p<\infty} \mathcal{H}_{w}^{p}$, and there exists $c>0$, such that the function $e^{c|\mathcal{B} g|}$ is integrable on $\mathbb{D}^{\infty}$, with respect to $d \mu_{w}$.

\section{Compactness}

We now present a little oh version of Theorem 1 .

If the symbol is a vector of the standard orthonormal basis of $\mathcal{H}_{w}^{2}$, that is

$$
g(s)=e_{w, n}(s):=w_{n}^{1 / 2} n^{-s}
$$

the operator $T_{g}^{*} T_{g}$ is diagonal, and its eigenvalues

$$
\lambda_{n, k}^{2}=\frac{w_{n} w_{k}}{w_{n k}}\left(\frac{\log n}{\log n+\log k}\right)^{2}
$$

tend to 0 as $k \rightarrow+\infty$. Thus $T_{g}$ is compact. It follows that every Dirichlet polynomial generates a compact Volterra operator on $\mathcal{H}_{w}^{2}$.

\subsection{Case when $\mathrm{Bg}$ depends on a finite number of variables}

We approximate a symbol $g$ which is in $\operatorname{Bloch}_{0}\left(\mathbb{C}_{0}\right) \cap \mathcal{D}_{d}$ by a Dirichlet polynomial $P$ in the $\operatorname{Bloch}\left(\mathbb{C}_{0}\right)$-norm. From Theorem $1(\mathrm{a}), T_{g}$ is approximated in the operator norm by the compact operator $T_{P}$.

Theorem 2 If $g$ is in Bloch $\left(\mathbb{C}_{0}\right) \cap \mathcal{D}_{d}$, then $T_{g}$ is compact on $\mathcal{H}_{w}^{2}$.

\subsection{Sufficient/necessary conditions for compactness}

In general, if the symbol $g(s)=\sum_{n \geq 2} b_{n} n^{-s}$ satisfies an inequality of the form $\left\|T_{g}\right\|_{\mathcal{L}\left(\mathcal{H}_{w}^{2}\right)}^{2} \leq \sum_{n \geq 2}\left|b_{n}\right|^{2} W(n)<\infty$, we approximate $T_{g}$ in the operator norm by the compact operator $T_{S_{N}}$. Therefore, $T_{g}$ is compact (see [13]).

The little oh version of Theorem 1 is related to the properties of $\operatorname{VMOA}\left(\mathbb{C}_{0}\right) \cap \mathcal{D}$, and with the concept of vanishing Carleson measures.

Theorem 3 Let $g$ be in $\mathcal{D}$.

(1) If $g$ is in $\operatorname{VMOA}\left(\mathbb{C}_{0}\right) \cap \mathcal{D}$, then $T_{g}$ is compact on $\mathcal{H}_{w}^{2}$.

(2) If $T_{g}$ is compact on $\mathcal{H}_{w}^{2}$, then $g$ is in Bloch $\left(\mathbb{C}_{1 / 2}\right)$. 
Proof In order to prove (1), we use that $\operatorname{VMOA}\left(\mathbb{C}_{0}\right) \cap \mathcal{D}$ is the closure of Dirichlet polynomials in $B M O A\left(\mathbb{C}_{0}\right)$ (see [13]), and that, from Theorem 1 , we have $\left\|T_{g}\right\|_{\mathcal{L}\left(\mathcal{H}_{w}^{2}\right)} \lesssim\|g\|_{B M O A\left(\mathbb{C}_{0}\right)}$.

Recall that $\mathcal{H}_{w}^{2}$ is embedded in $A_{i, \delta}\left(\mathbb{C}_{1 / 2}\right), \delta=\delta(w)$ being defined in (2.5). Assume that $T_{g}$ is compact on $\mathcal{H}_{w}^{2}$, and consider the measure

$$
d v_{g}(s)=\left|g^{\prime}(s)\right|^{2} \frac{\left(\sigma-\frac{1}{2}\right)^{\delta+1}}{\left|s+\frac{1}{2}\right|^{2(\delta+1)}} d V(s)
$$

Let $\left(f_{k}\right)_{k}$ be a weakly compact sequence in $\mathcal{H}_{w}^{2}$. Formula (3.1), and Lemma 1 imply that

$$
\int_{\mathbb{C}_{1 / 2}}\left|f_{k}(s)\right|^{2} d \nu_{g}(s) \asymp\left\|T_{g} f_{k}\right\|_{A_{i, \delta}\left(\mathbb{C}_{1 / 2}\right)}^{2} \lesssim\left\|T_{g} f_{k}\right\|_{\mathcal{H}_{w}^{2}}^{2} .
$$

By the compactness of $T_{g}, v_{g}$ is a vanishing Carleson measure for $A_{i, \delta}\left(\mathbb{C}_{1 / 2}\right)$, with

$$
\lim _{k \rightarrow \infty} \int_{\mathbb{C}_{1 / 2}}\left|f_{k}(s)\right|^{2} d v_{g}(s)=0
$$

Now, $g$ is in $\operatorname{Bloch}_{0}\left(\mathbb{C}_{1 / 2}\right)$, by the characterization of vanishing Carleson measures (Lemma 5).

\section{Membership in Schatten classes}

Let $g$ be a non constant symbol. As in the case of $\mathcal{H}^{2}$, the Volterra operator $T_{g}$ on $\mathcal{H}_{w}^{2}$ does not belong to any Schatten class.

Theorem 4 If the Dirichlet series $g(s)=\sum_{n \geq 2} b_{n} n^{-s}$ is not 0 , then $T_{g}: \mathcal{H}_{w}^{2} \rightarrow \mathcal{H}_{w}^{2}$ is not in the Schatten class $\mathcal{S}_{p}$, for any $0<p<\infty$.

Proof Recall that $\left(e_{w, n}\right)_{n}$ is an orthonormal basis of $\mathcal{H}_{w}^{2}$. We follow the reasoning of Theorem 7.2 [13]. Using that $w_{N n} \lesssim w_{N} w_{n}$, we see that, for $N \geq n$,

$$
\left\|T_{g} e_{w, n}\right\|_{\mathcal{H}_{w}^{2}}^{2}=\sum_{k=2}^{+\infty} \frac{\left|b_{k}\right|^{2}(\log k)^{2}}{(\log (k n))^{2}} \frac{w_{n}}{w_{k n}} \geq \frac{\left|b_{N}\right|^{2}(\log N)^{2}}{(\log (N n))^{2}} \frac{w_{n}}{w_{N n}} \gtrsim \frac{\left|b_{N}\right|^{2}(\log N)^{2}}{(2 \log n)^{2}} \frac{1}{w_{N}}
$$

For $p \geq 2$, we obtain

$$
\left\|T_{g}\right\|_{\mathcal{S}_{p}}^{p} \geq \sum_{n=N}^{+\infty}\left\|T_{g} e_{w, n}\right\|_{\mathcal{H}_{w}^{2}}^{p}=+\infty
$$

Therefore $T_{g}$ is not in $\mathcal{S}_{p}$ for $p \geq 2$, neither for $0<p<\infty$. 


\section{Examples}

In this section, we study the boundedness of $T_{g}$ on $\mathcal{H}_{w}^{2}$, for specific symbols $g$. We consider fractional primitives of translates of the weighted Zeta function $Z_{w}$ and homogeneous symbols, which are the counterparts of the symbols presented in [13] in the $\mathcal{H}^{2}$ setting. The techniques of proof, as well as the results are similar to theirs, and we omit the details.

\subsection{Fractional primitives of translates of $Z_{w}$}

Proposition 4 With the notation of (2.5), take $1 / 2 \leq a<1,2 \gamma>\delta(w)-1$. If

$$
g(s)=\sum_{n=2}^{\infty} w_{n} \frac{n^{-a}}{(\log n)^{\gamma+1}} n^{-s}
$$

then $T_{g}$ is unbounded on $\mathcal{H}_{w}^{2}$.

Proof Abel summation and the Chebyshev estimate induce that $g$ is in $\mathcal{H}_{w}^{2}$. If $f(s)=$ $\sum_{n=1}^{\infty} a_{n} n^{-s}$, and $g(s)=\sum_{n=2}^{\infty} \frac{b_{n}}{\log n} n^{-s}$, we set $A_{n}=\sum_{k \mid n} a_{n / k} b_{k}$, so that

$$
\left\|\tilde{T}_{g} f\right\|_{\mathcal{H}_{w}^{2}}^{2}=\sum_{n=2}^{\infty} \frac{1}{\left(w_{n} \log n\right)^{2}} A_{n}^{2} .
$$

We adapt the test functions of [13], and take $f_{J}(s)=\prod_{j=1}^{J}\left(1+w_{2}^{1 / 2} p_{j}^{-s}\right)$, for $J \geq 1$. By construction, it satisfies $\left\|f_{J}\right\|_{\mathcal{H}_{w}^{2}} \asymp 2^{J / 2}$. Now, for $\mathcal{J}$ a non-empty subset of $\{1, \ldots, J\}$, we set $n_{\mathcal{J}}=\prod_{j \in \mathcal{J}} p_{j}$, and observe that

$$
A_{n_{\mathcal{J}}}=\sum_{1 \leq k \leq|\mathcal{J}|,\left\{p_{j_{1}}, \ldots, p_{j_{k}}\right\} \subset \mathcal{J}} w_{2}^{\frac{|\mathcal{J}|-k}{2}}\left[\log \left(p_{j_{1}} \cdots p_{j_{k}}\right)\right]^{-\gamma} w_{2}^{k}\left(p_{j_{1}} \cdots p_{j_{k}}\right)^{-a}+w_{2}{ }^{\frac{|\mathcal{J}|}{2}}
$$

First assume that $\gamma \geq 0$. From the prime number Theorem, we obtain that

$$
A_{n_{\mathcal{J}}} \gtrsim w_{2}^{\frac{|\mathcal{J}|}{2}}[J \log J]^{-\gamma}\left[1+\sum_{1 \leq k \leq|\mathcal{J}|,\left\{p_{j_{1}}, \ldots, p_{j_{k}}\right\} \subset \mathcal{J}} w_{2}^{k / 2}\left(p_{j_{1}} \cdots p_{j_{k}}\right)^{-a}\right] .
$$

Therefore, it follows again from the prime number Theorem that

$$
\begin{aligned}
& \left\|\tilde{T}_{g} f_{J}\right\|_{\mathcal{H}_{w}^{2}}^{2} \gtrsim \sum_{\mathcal{J} \subset\{1, \ldots, J\},|\mathcal{J}| \geq J / 2} \frac{1}{\left(\log n_{\mathcal{J}}\right)^{2}}[J \log J]^{-2 \gamma} \prod_{j \in \mathcal{J}}\left(1+w_{2}^{1 / 2} p_{j}^{-a}\right)^{2} \\
& \gtrsim 2^{J-1}[J \log J]^{-2 \gamma} \min _{|\mathcal{J}| \geq J / 2} \frac{1}{\left(\log n_{\mathcal{J}}\right)^{2}} \prod_{j \in \mathcal{J}}\left(1+w_{2}^{1 / 2} p_{j}^{-a}\right)^{2}
\end{aligned}
$$




$$
\gtrsim e^{c J^{1-a}(\log J)^{-a}}\left\|f_{J}\right\|_{\mathcal{H}_{w}^{2}}^{2}
$$

for some constant $c>0$, and $T_{g}$ is unbounded. The case when $\gamma<0$ is similar.

\subsection{Homogeneous symbols}

An m-homogeneous Dirichlet series has the form

$$
g(s)=\sum_{\Omega(n)=m} b_{n} n^{-s} .
$$

We extend Theorem 4.2 in [13] to the spaces $\mathcal{H}_{w}^{2}$.

Proposition 5 There exist weights $W_{m}(n)$ such that for $g(s)=\sum_{\Omega(n)=m} b_{n} n^{-s}$,

$$
\left\|T_{g}\right\|_{\mathcal{L}\left(\mathcal{H}_{w}^{2}\right)} \leq\left(\sum_{\Omega(n)=m}\left|b_{n}\right|^{2} W_{m}(n)\right)^{1 / 2}
$$

Precisely, there exist absolute constants $C_{m}$ for which

$$
W_{m}(n)= \begin{cases}C_{1} & \text { for } m=1, \\ C_{2} \frac{\log n}{\log _{2} n} & \text { for } m=2, \\ C_{m} \frac{n \frac{m-2}{m}}{(\log n)^{m-2}} & \text { for } m \geq 3 .\end{cases}
$$

Moreover, when $m=2, \log _{2} n$ cannot be replaced in (7.1) by $\left(\log _{2} n\right)^{1+\varepsilon}$ for any $\varepsilon>0$.

Proof If a linear symbol $(m=1) g(s)=\sum_{p \in \mathbb{P}} b_{p} p^{-s}$ belongs to $\mathcal{H}^{2}$, we observe that $\|g\|_{\mathcal{H}^{2}}^{2}=2^{\beta}\|g\|_{\mathcal{B}_{\beta}^{2}}^{2}=(\beta+1)\|g\|_{\mathcal{A}_{\beta}^{2}}^{2}$. Hence, it follows from Theorem 4.1 in [13] and Lemma 9 that $T_{g}$ is bounded on $\mathcal{H}_{w}^{2}$ and $\left\|T_{g}\right\|_{\mathcal{L}\left(\mathcal{H}_{w}^{2}\right)} \leq\left\|T_{g}\right\|_{\mathcal{L}\left(\mathcal{H}^{2}\right)}$. One can choose $C_{1}=\max \left((\beta+1)^{-1}, 2^{-\beta}\right)$.

(7.1) is a consequence of Theorem 4.2 in [13] and Lemma 9. We now prove the sharpness of the factor $\log _{2} n$. We assume that for some $\varepsilon>0$, every 2-homogeneous Dirichlet series $g$ satisfies

$$
\left\|T_{g}\right\|_{\mathcal{L}\left(\mathcal{H}_{w}^{2}\right)} \leq C_{2}\left(\sum_{\Omega(n)=m}\left|b_{n}\right|^{2} \frac{\log n}{\left(\log _{2} n\right)^{1+\varepsilon}}\right)^{1 / 2}
$$


For $x$ a large real number, and $q \sim e^{x}$ a prime number, the symbol considered in [13] is

$$
g_{x}(s)=\sum_{x / 2<p \leq x} \frac{\left(\log _{2}(p q)\right)^{1+\varepsilon / 2}}{p}(p q)^{-s} .
$$

We take as test functions

$$
f_{x}(s)=\sum_{n=1}^{+\infty} a_{n} n^{-s}=\prod_{x / 2<p \leq x}\left(1+w_{2}^{1 / 2} p^{-s}\right) .
$$

If $S_{x}$ denotes the set of square-free integers generated by the primes $x / 2<p \leq x$, we have $\left\|f_{x}\right\|_{\mathcal{H}_{w}^{2}}^{2} \asymp\left|S_{x}\right|=2^{N(x)}$, where $N(x):=\pi(x)-\pi(x / 2)$. Now,

$$
\frac{\left\|T_{g_{x}} f_{x}\right\|_{\mathcal{H}_{w}^{2}}^{2}}{\left\|f_{x}\right\|_{\mathcal{H}_{w}^{2}}^{2}} \gtrsim \frac{1}{\left|S_{x}\right|} \sum_{n \in S_{x}} w_{n q}^{-1}(\log (n q))^{-2}\left|\sum_{p q \mid n q} \log (p q) \frac{\left(\log _{2}(p q)\right)^{1+\varepsilon / 2}}{p} a_{n / p}\right|^{2} .
$$

If $n \in S_{x}$, and $p \mid n$, we have $a_{n / p}=w_{2}^{\frac{1}{2}[\omega(n)-1]}, w_{n}=w_{2}^{\omega(n)}$, and $w_{n q}=w_{n} w_{q}$. Thus,

$$
\frac{\left\|T_{g_{x}} f_{x}\right\|_{\mathcal{H}_{w}^{2}}^{2}}{\left\|f_{x}\right\|_{\mathcal{H}_{w}^{2}}^{2}} \gtrsim \frac{1}{\left|S_{x}\right|} \frac{(\log x)^{2+\varepsilon}}{x^{2}} \sum_{n \in S_{x}} \omega(n)^{2} .
$$

Now $\sum_{n \in S_{x}} \omega(n)^{2}=\sum_{k=1}^{N(x)}\left(\begin{array}{c}N(x) \\ k\end{array}\right) k^{2} \asymp N(x)^{2} 2^{N(x)}$, and (7.2) does not hold, due to

$$
\frac{\left\|T_{g_{x}} f_{x}\right\|_{\mathcal{H}_{w}^{2}}}{\left\|f_{x}\right\|_{\mathcal{H}_{w}^{2}}} \gtrsim(\log x)^{\varepsilon} .
$$

We will exhibit an homogeneous symbol $g$ which is in $\mathcal{H}_{w}^{2} \cap \operatorname{Bloch}_{0}\left(\mathbb{C}_{1 / 2}\right)$, but not in $\mathcal{X}_{w}$. In fact, we observe that $g$ is in every $\mathcal{H}_{w}^{p}$.

Lemma 10 If $g$ is an m-homogeneous Dirichlet series in $\mathcal{H}_{w}^{2}$, then $g$ is in $\cap_{0<p<\infty} \mathcal{H}_{w}^{p}$ and, for any $0<p<\infty$, there exists $c=c(m, p)$ such that

$$
\|g\|_{\mathcal{H}_{w}^{p}} \leq c\|g\|_{\mathcal{H}_{w}^{2}}
$$

Proof It is enough to consider the case $p \geq 2$. We first prove the inequality for $p=2^{k}$, $k$ being a positive integer, by an induction argument.

Obviously, it holds for $k=1$. 
Our proof is inspired of Lemma 8 in [27]. For any integer $m$, there exists a constant $C(m)$, such that $\max \left(w_{n}, d(n)\right) \leq C(m)$, whenever $\Omega(n)=m$.

If $f(s)=\sum_{n} a_{n} n^{-s}$ is $m$-homogeneous, then $f^{2}(s)=\sum_{n} b_{n} n^{-s}$ is $2 m$ homogeneous, and $\left|b_{n}\right|^{2} \leq d(n) \sum_{k \mid n}\left|a_{k}\right|^{2}\left|a_{n / k}\right|^{2}$. Since $w_{n} \geq \sqrt{w_{k}} \sqrt{w_{n / k}}$,

$$
\begin{aligned}
\|f\|_{\mathcal{H}_{w}^{4}}^{4} & =\left\|f^{2}\right\|_{\mathcal{H}_{w}^{2}}^{2} \leq \sum_{\Omega(n)=2 m} d(n) w_{n}^{-1}\left(\sum_{k \mid n}\left|a_{k}\right|^{2}\left|a_{n / k}\right|^{2}\right) \\
& \leq C(2 m) \sum_{\Omega(n)=2 m}\left(\sum_{k \mid n} \frac{\left|a_{k}\right|^{2}}{\sqrt{w_{k}}} \frac{\left|a_{n / k}\right|^{2}}{\sqrt{w_{n / k}}}\right) \\
& =C(2 m)\left(\sum_{k} \frac{\left|a_{k}\right|^{2}}{\sqrt{w_{k}}}\right)^{2} \leq C(2 m) C(m)\|f\|_{\mathcal{H}_{w}^{2}}^{4} .
\end{aligned}
$$

Now, suppose that, for some $k$, an $m$-homogeneous Dirichlet series $h$ satisfies

$$
\|h\|_{\mathcal{H}_{w}^{2^{k}}}^{2^{k}} \leq K(m, k)\|h\|_{\mathcal{H}_{w}^{2}}^{2^{k}} \text { for any } m
$$

We obtain that

$$
\begin{aligned}
\|f\|_{\mathcal{H}_{w}^{2^{k+1}}}^{2^{k+1}} & =\left\|f^{2}\right\|_{\mathcal{H}_{w}^{2^{k}}}^{2^{k}} \leq K(2 m, k)\left\|f^{2}\right\|_{\mathcal{H}_{w}^{2}}^{2^{k}}=K(2 m, k)\|f\|_{\mathcal{H}_{w}^{4}}^{2^{k+1}} \\
& \leq K(2 m, k)\left[C(2 m) C(m)\|f\|_{\mathcal{H}_{w}^{2}}^{4}\right]^{2^{k-1}} .
\end{aligned}
$$

For general $p,(7.3)$ is a consequence of Hölder's inequality.

For our construction, we need two technical Lemmas.

Lemma 11 Assume that $0<\delta<1$ and $0<\eta$. For $j=1 . .3$, we set $h_{j}(s)=$ $\sum_{p \geq 3} \alpha_{j, p} p^{-s}$, where

$$
\alpha_{1, p}=\left(\log _{2} p\right)^{-\delta}, \alpha_{2, p}=\log _{2} p, \alpha_{3, p}=\log p\left(\log _{2} p\right)^{-\eta} .
$$

For a real number $\sigma>1$, set $\sigma^{\prime}:=\frac{1}{\sigma-1}$. Then we have

$$
h_{1}(\sigma) \asymp\left(\log \sigma^{\prime}\right)^{1-\delta} ; h_{2}(\sigma) \asymp \log _{2}\left(\sigma^{\prime}\right) ; h_{3}(\sigma) \asymp \sigma^{\prime}\left(\log \sigma^{\prime}\right)^{-\eta}, \text { as } \sigma \rightarrow 1^{+} \text {. }
$$

Proof These asymptotics will follow from computations inspired by [4,20]. Recall that

$$
A_{1}(t):=\sum_{3 \leq p \leq t} \frac{1}{p}=\log _{2} t+O(1) .
$$


Setting $f_{1}(t)=\frac{t^{-(\sigma-1)}}{\left(\log _{2} t\right)^{\delta}}$, we have

$$
\begin{aligned}
h_{1}(\sigma) & =\sum_{p \geq 3} \frac{p^{-(\sigma-1)}}{p\left(\log _{2} p\right)^{\delta}}=-\int_{3}^{+\infty} A_{1}(t) f_{1}^{\prime}(t) d t+O(1) \\
& \asymp(\sigma-1) \int_{3}^{+\infty}\left(\log _{2} t\right)^{1-\delta} t^{-\sigma} d t \\
& =(\sigma-1)\left(\int_{\log 3}^{\sigma^{\prime}}+\int_{\sigma^{\prime}}^{+\infty}\right)(\log x)^{1-\delta} e^{-(\sigma-1) x} d x .
\end{aligned}
$$

Using integration by parts (for the first integral), and a change of variable (for the second one), we obtain

$$
\begin{aligned}
h_{1}(\sigma) & \asymp(\sigma-1) \int_{\log 3}^{\sigma^{\prime}}(\log x)^{1-\delta} d x+\int_{1}^{+\infty}\left(\log y+\log \sigma^{\prime}\right)^{1-\delta} e^{-y} d y \\
& \asymp(\sigma-1)\left[x(\log x)^{1-\delta}\right]_{x=\log 3}^{x=\sigma^{\prime}}+\int_{1}^{+\infty}\left[(\log y)^{1-\delta}+\left(\log \sigma^{\prime}\right)^{1-\delta}\right] e^{-y} d y \\
& \asymp\left(\log \sigma^{\prime}\right)^{1-\delta} .
\end{aligned}
$$

The functions $h_{2}$ and $h_{3}$ are handled similarly. For $x \geq 3$, summation by parts and (7.5) induce that,

$$
A_{2}(x):=\sum_{3 \leq p \leq x} \frac{1}{p \log _{2} p}=\frac{A_{1}(x)}{\log _{2} x}+\int_{3}^{x} \frac{A_{1}(t)}{t \log t\left(\log _{2} t\right)^{2}} d t+O(1) \asymp \log _{3} x .
$$

Set $f_{2}(t):=t^{-(\sigma-1)}$. Then,

$$
\begin{aligned}
h_{2}(\sigma) & \asymp-\int_{3}^{+\infty} A_{2}(t) f_{2}^{\prime}(t) d t+O(1) \asymp(\sigma-1) \int_{3}^{+\infty}\left(\log _{3} t\right) t^{-\sigma} d t \\
& =(\sigma-1)\left(\int_{\log 3}^{e \sigma^{\prime}}+\int_{e \sigma^{\prime}}^{+\infty}\right)\left(\log _{2} x\right) e^{-(\sigma-1) x} d x
\end{aligned}
$$

Now

$$
\begin{aligned}
(\sigma-1) \int_{\log 3}^{e \sigma^{\prime}}\left(\log _{2} x\right) e^{-(\sigma-1) x} d x & \asymp(\sigma-1) \int_{\log 3}^{e \sigma^{\prime}}\left(\log _{2} x\right) d x \\
& \leq(\sigma-1) e \sigma^{\prime}\left(\log _{2}\left(e \sigma^{\prime}\right)\right) \lesssim \log _{2} \sigma^{\prime}
\end{aligned}
$$

We perform a change of variable in the integral over $\left[e \sigma^{\prime},+\infty\right)$.

$$
I_{2,2}:=(\sigma-1) \int_{e \sigma^{\prime}}^{+\infty}\left(\log _{2} x\right) e^{-(\sigma-1) x} d x=\int_{e}^{+\infty}\left[\log \left(\log y+\log \sigma^{\prime}\right)\right] e^{-y} d y
$$




$$
\geq\left(\log _{2} \sigma^{\prime}\right) \int_{e}^{+\infty} e^{-y} d y \gtrsim \log _{2} \sigma^{\prime} .
$$

Since $\log (a+b) \leq \log a \log b+1$, for $a \geq e$ and $b \geq e$, we obtain

$$
I_{2,2} \leq \int_{e}^{+\infty}\left[\left(\log _{2} y\right)\left(\log _{2} \sigma^{\prime}\right)+1\right] e^{-y} d y \lesssim \log _{2} \sigma^{\prime},
$$

and $I_{2,2} \asymp \log _{2} \sigma^{\prime}$. It follows that $h_{2}(\sigma) \asymp \log _{2} \sigma^{\prime}$.

We now focus on $h_{3}$. By Mertens' first Theorem, $A_{3}(x):=\sum_{3 \leq p \leq x} \frac{\log p}{p}=$ $\log x+O(1)$, and putting $f_{3}(t):=t^{-(\sigma-1)}\left(\log _{2} t\right)^{-\eta}$, we see that

$$
\begin{aligned}
h_{3}(\sigma) & =-\int_{3}^{+\infty} A_{3}(t) f_{3}^{\prime}(t) d t+O(1) \\
& \asymp(\sigma-1) \int_{3}^{+\infty}(\log t) t^{-\sigma}\left(\log _{2} t\right)^{-\eta} d t \\
& \asymp(\sigma-1)\left(\int_{\log 3}^{\sigma^{\prime}}+\int_{\sigma^{\prime}}^{+\infty}\right) x e^{-(\sigma-1) x}(\log x)^{-\eta} d x .
\end{aligned}
$$

Integration by parts gives that

$$
\begin{aligned}
I_{3,1} & :=(\sigma-1) \int_{\log 3}^{\sigma^{\prime}} x e^{-(\sigma-1) x}(\log x)^{-\eta} d x \\
& \asymp(\sigma-1) \int_{\log 3}^{\sigma^{\prime}} x(\log x)^{-\eta} d x \asymp \sigma^{\prime}\left(\log \sigma^{\prime}\right)^{-\eta} .
\end{aligned}
$$

Next, (7.4) is a consequence of

$$
\begin{aligned}
I_{3,2} & :=(\sigma-1) \int_{\sigma^{\prime}}^{+\infty} x e^{-(\sigma-1) x}(\log x)^{-\eta} d x \\
& =\frac{1}{\sigma-1} \int_{1}^{+\infty} y e^{-y}\left(\log y+\log \sigma^{\prime}\right)^{-\eta} d y \\
& \lesssim \sigma^{\prime} \int_{1}^{+\infty} \frac{y e^{-y}}{\left(\log \sigma^{\prime}\right)^{\eta}} d y .
\end{aligned}
$$

Lemma 12 If $2 \eta>1$ and $\delta+\eta>1$, we have

$$
\begin{aligned}
S:= & \sum_{p_{1}, p_{2}, p_{3} \in \mathbb{P}, p_{j} \geq 3} \frac{1}{p_{1} p_{2} p_{3}\left(\log _{2} p_{1}\right)^{2 \delta}\left(\log _{2} p_{2}\right)^{2}} \times \\
& \frac{\left(\log p_{3}\right)^{2}}{\left(\log _{2} p_{3}\right)^{2 \eta}\left(\log \left(p_{1} p_{2} p_{3}\right)\right)^{2}}<\infty .
\end{aligned}
$$


Proof For $p_{1}, p_{2} \geq 3$, we set $L:=\log \left(p_{1} p_{2}\right)$ and $S_{3}\left(p_{1}, p_{2}\right):=$ $\sum_{p_{3}} \frac{\left(\log p_{3}\right)^{2}}{p_{3}\left(\log _{2} p_{3}\right)^{2 \eta}\left(\log p_{3}+L\right)^{2}}$. Then, we have

$$
S=\sum_{p_{1}, p_{2}, p_{3}} \frac{1}{p_{1} p_{2}\left(\log _{2} p_{1}\right)^{2 \delta}\left(\log _{2} p_{2}\right)^{2}} S_{3}\left(p_{1}, p_{2}\right)
$$

Under the condition $2 \eta>1$, the sum $S_{3}\left(p_{1}, p_{2}\right)$ converges, and

$$
\begin{aligned}
S_{3}\left(p_{1}, p_{2}\right) & =-\int_{3}^{+\infty} A_{1}(t) \frac{d}{d t}\left[\frac{(\log t)^{2}}{\left(\log _{2} t\right)^{2 \eta}(\log t+L)^{2}}\right] d t+\frac{O(1)}{L^{2}} \\
& \lesssim \frac{O(1)}{L^{2}}+\int_{3}^{+\infty} \frac{\log t}{t\left(\log _{2} t\right)^{2 \eta}(\log t+L)^{2}} d t \\
& =\frac{O(1)}{L^{2}}+\left(\int_{\log 3}^{L}+\int_{L}^{+\infty}\right) \frac{x d x}{(\log x)^{2 \eta}(x+L)^{2}} .
\end{aligned}
$$

Integration by parts gives

$$
I_{3,1}:=\int_{\log 3}^{L} \frac{x d x}{(\log x)^{2 \eta}(x+L)^{2}} \asymp \frac{1}{L^{2}} \int_{\log 3}^{L} \frac{x d x}{(\log x)^{2 \eta}} \asymp(\log L)^{-2 \eta} .
$$

We handle the second integral via a change of variable:

$$
\begin{aligned}
I_{3,2} & :=\int_{L}^{+\infty} \frac{x d x}{(\log x)^{2 \eta}(x+L)^{2}}=\left(\int_{1}^{L}+\int_{L}^{+\infty}\right) \frac{y d y}{(1+y)^{2}(\log y+\log L)^{2 \eta}} \\
& \lesssim \frac{1}{(\log L)^{2 \eta}} \int_{1}^{L} \frac{d y}{y}+\int_{L}^{+\infty} \frac{d y}{y(\log y)^{2 \eta}} \asymp(\log L)^{1-2 \eta} .
\end{aligned}
$$

Therefore

$$
S_{3}\left(p_{1}, p_{2}\right) \lesssim(\log L)^{1-2 \eta}, L=\log \left(p_{1} p_{2}\right)
$$

We next put $M=\log p_{1}$, and deal with

$$
S_{2}\left(p_{1}\right):=\sum_{p_{2}} \frac{1}{p_{2}\left(\log _{2} p_{2}\right)^{2}} S_{3}\left(p_{1}, p_{2}\right) \lesssim \sum_{p} \frac{1}{p\left(\log _{2} p\right)^{2}[\log (\log p+M)]^{2 \eta-1}}
$$

With the notation $f_{2}(t):=\left[\left(\log _{2} t\right)^{2}[\log (\log t+M)]^{2 \eta-1}\right]^{-1}$, we obtain that

$$
S_{2}\left(p_{1}\right)=\frac{O(1)}{(\log M)^{2 \eta-1}}-\int_{3}^{+\infty} A_{1}(t) f_{2}^{\prime}(t) d t \lesssim \frac{O(1)}{(\log M)^{2 \eta-1}}+I_{2,1}+I_{2,2},
$$


where

$$
\begin{aligned}
I_{2,1} & :=\int_{3}^{+\infty} \frac{d t}{t \log t\left(\log _{2} t\right)^{2}[\log (\log t+M)]^{2 \eta-1}} \\
I_{2,2} & :=\int_{3}^{+\infty} \frac{d t}{t\left(\log _{2} t\right)(\log t+M)[\log (\log t+M)]^{2 \eta}}
\end{aligned}
$$

We derive

$$
\begin{aligned}
I_{2,1} & =\left(\int_{\log 3}^{M}+\int_{M}^{+\infty}\right) \frac{d x}{x(\log x)^{2}[\log (x+M)]^{2 \eta-1}} \\
\lesssim & \frac{1}{[\log M]^{2 \eta-1}} \int_{\log 3}^{M} \frac{d x}{x(\log x)^{2}} \\
& +(\log M)^{1-2 \eta} \int_{M}^{+\infty} \frac{d x}{x(\log x)^{2}} \lesssim(\log M)^{1-2 \eta}
\end{aligned}
$$

The second integral is estimated in the same way:

$$
\begin{aligned}
I_{2,2} & =\left(\int_{\log 3}^{M}+\int_{M}^{+\infty}\right) \frac{d x}{(x+M)(\log x)[\log (x+M)]^{2 \eta}} \\
& \lesssim \frac{1}{M(\log M)^{2 \eta}} \int_{\log 3}^{M} \frac{d x}{\log x}+\frac{1}{(\log M)^{2 \eta-1}} \int_{M}^{+\infty} \frac{d x}{x(\log x)^{2}} \\
\asymp & \frac{1}{M(\log M)^{2 \eta}}\left(\left[\frac{x}{\log x}\right]_{x=\log 3}^{x=M}+\int_{\log 3}^{M} \frac{x^{2}}{2} \frac{(\log x)^{-2}}{x} d x\right) \\
& +\frac{1}{(\log M)^{2 \eta}} \asymp \frac{1}{(\log M)^{2 \eta}} .
\end{aligned}
$$

Therefore, we have

$$
S_{2}\left(p_{1}\right) \lesssim \frac{1}{(\log M)^{2 \eta-1}}, M=\log p_{1}
$$

It follows that

$$
S \lesssim \sum_{p_{1}} \frac{1}{p_{1}\left(\log _{2} p_{1}\right)^{2 \delta}} S_{2}\left(p_{1}\right) \lesssim \sum_{p \geq 3} \frac{1}{p\left(\log _{2} p\right)^{\varepsilon}}, \varepsilon:=2 \delta+2 \eta-1
$$

Again, partial summation gives that when $\varepsilon>1$, 


$$
\sum_{3 \leq p} \frac{1}{p\left(\log _{2} p\right)^{\varepsilon}} \asymp \varepsilon \int_{3}^{+\infty} \frac{\log _{2} t}{t(\log t)\left(\log _{2} t\right)^{\varepsilon+1}} d t<\infty
$$

Proposition 6 There exists a 3-homogeneous function $g$ which is in $\left(\cap_{0<p<\infty} \mathcal{H}_{w}^{p}\right) \cap$ Bloch $\left(\mathbb{C}_{1 / 2}\right)$, such that $T_{g}$ is unbounded on $\mathcal{H}_{w}^{2}$.

Proof Using Lemma 11, we see that, if $g^{\prime}=-\left(h_{1} h_{2} h_{3}\right)_{\frac{1}{2}}, g^{\prime}$ is convergent on $\mathbb{C}_{1 / 2}$, and its estimate near the line $\Re s=\frac{1}{2}$ is determined by the behavior of the functions $h_{j}$ near the line $\Re s=1$. Then $g$ is in $\operatorname{Bloch}_{0}\left(\mathbb{C}_{1 / 2}\right)$, because of

$$
\left|g^{\prime}(\sigma)\right| \asymp \frac{1}{\sigma-\frac{1}{2}}\left(\log \frac{1}{\sigma-\frac{1}{2}}\right)^{1-\delta-\eta}\left(\log _{2} \frac{1}{\sigma-\frac{1}{2}}\right), \text { as } \sigma \rightarrow 1 / 2^{+} \text {. }
$$

On another hand, the 3-homogeneous function

$$
g(s)=\sum_{n} b_{n} n^{-s}=\sum_{p_{1}, p_{2}, p_{3}} \frac{\alpha_{1, p_{1} \alpha_{2, p_{2}} \alpha_{3, p_{3}}}}{\log \left(p_{1} p_{2} p_{3}\right)}\left(p_{1} p_{2} p_{3}\right)^{-s}
$$

is in $\mathcal{H}_{w}^{2}$ by Lemma 12 , since $\|g\|_{\mathcal{H}_{w}^{2}}^{2}=\sum_{n}\left|b_{n}\right|^{2} w_{n}^{-1} \asymp \sum_{n}\left|b_{n}\right|^{2} \asymp S<\infty$.

By Lemma 10, $g$ is in $\cap_{0<p<\infty} \mathcal{H}_{w}^{p}$.

It remains to prove that $T_{g}$ is unbounded on $\mathcal{H}_{w}^{2}$. We again choose as test functions (cf the proof of Proposition 5)

$$
f_{x}(s):=\prod_{\frac{x}{2}<p \leq x}\left(1+w_{2}^{1 / 2} p^{-s}\right)=\sum_{n \geq 1} a_{n} n^{-s} .
$$

$S_{x}$ is the set of square free integers generated by $\frac{x}{2}<p \leq x$. Set $V_{x}=$ $\left\{n \in S_{x}, \omega(n) \geq \frac{N(x)}{2}\right\}$.

For $n \in V_{x}$, set

$$
A_{n}:=\sum_{p_{1} p_{2} p_{3} \mid n} b_{p_{1} p_{2} p_{3}}\left(\log \left(p_{1} p_{2} p_{3}\right)\right) a_{\frac{n}{p_{1} p_{2} p_{3}}}
$$

The coefficients in $A_{n}$ satisfy

$$
b_{p_{1} p_{2} p_{3}}\left(\log \left(p_{1} p_{2} p_{3}\right)\right) \gtrsim \frac{\log x}{x^{3 / 2}\left(\log _{2} x\right)^{\eta+\delta+1}} .
$$

Since $\left\|f_{x}\right\|_{\mathcal{H}_{w}^{2}}^{2} \asymp\left|V_{x}\right|$, we see that

$$
\left\|T_{g} f_{x}\right\|_{\mathcal{H}_{w}^{2}}^{2} \geq \sum_{n \in V_{x}} w_{n}^{-1}(\log n)^{-2} A_{n}^{2}
$$




$$
\begin{aligned}
& \gtrsim \sum_{n \in V_{x}} w_{2}^{-\omega(n)}(\omega(n) \log x)^{-2} \times \\
& {\left[\frac{\log x}{x^{3 / 2}\left(\log _{2} x\right)^{\eta+\delta+1}}\left(\begin{array}{c}
\omega(n) \\
3
\end{array}\right)\left(w_{2}^{1 / 2}\right)^{\omega(n)-3}\right]^{2}} \\
& \gtrsim\left\|f_{x}\right\|_{\mathcal{H}_{w}^{2}}^{2}\left(\frac{x}{\log x}\right)^{4} \frac{1}{x^{3}\left(\log _{2} x\right)^{2(\delta+\eta+1)}},
\end{aligned}
$$

and the proof is complete.

\section{Comparison of $\mathcal{X}_{w}$ with other spaces of Dirichlet series}

The previous results enable us to derive some inclusions involving $\mathcal{X}_{w}$.

In the context of the unit disk, the space of symbols $g$ for which the Volterra operator $J_{g}(1.3)$ is bounded on $A_{\alpha}^{2}(\mathbb{D})$ is Bloch $(\mathbb{D})$. It coincides with the space of holomorphic $g$ such that the Hankel form (1.5) is bounded, and with the dual space of $A_{\alpha}^{1}(\mathbb{D})$.

We shall study the counterparts of these facts for $\mathcal{X}_{w}$.

\subsection{Bounded Hankel forms}

The Hilbert space $\mathcal{H}_{w}^{2}$ is equipped with the inner product $\langle., .\rangle_{\mathcal{H}_{w}^{2}}$. The Hankel form of symbol $g \in \mathcal{D}$ is defined on $\mathcal{H}_{w}^{2}$ by

$$
H_{g}(f h):=\langle f h, g\rangle_{\mathcal{H}_{w}^{2}} .
$$

We say that $H_{g}$ is bounded on $\mathcal{H}_{w}^{2} \times \mathcal{H}_{w}^{2}$ if there is a constant $C$ such that

$$
\left|H_{g}(f h)\right| \leq C\|f\|_{\mathcal{H}_{w}^{2}}\|h\|_{\mathcal{H}_{w}^{2}} \text { for } f, h \in \mathcal{H}_{w}^{2} \text {. }
$$

The weak product $\mathcal{H}_{w}^{2} \odot \mathcal{H}_{w}^{2}$ is the Banach space defined as the closure of all finite sums $F=\sum_{k} f_{k} h_{k}$, where $f_{k}, h_{k} \in \mathcal{H}_{w}^{2}$, under the norm

$$
\|F\|_{\mathcal{H}_{w}^{2} \odot \mathcal{H}_{w}^{2}}:=\inf \sum_{k}\left\|f_{k}\right\|_{\mathcal{H}_{w}^{2}}\left\|h_{k}\right\|_{\mathcal{H}_{w}^{2}}
$$

Here the infimum is taken over all finite representations of $F$ as $F=\sum_{k} f_{k} h_{k}$.

Let $\mathcal{Y}$ be a Banach space of Dirichlet series in which the space of Dirichlet polynomials $\mathcal{P}$ is dense. We say that a Dirichlet series $\phi$ is in the dual space $\mathcal{Y}^{*}$ if the linear functional induced by $\phi$ via the $\mathcal{H}_{w}^{2}$-pairing is bounded. In other words, $\phi \in \mathcal{Y}^{*}$ if and only if

$$
v_{\phi}(f)=\langle f, \phi\rangle_{\mathcal{H}_{w}^{2}}, f \in \mathcal{P}
$$


extends to a bounded functional on $\mathcal{Y}$.

From its definition, $H_{g}(8.1)$ is bounded on $\mathcal{H}_{w}^{2}$ if and only if $g \in\left(\mathcal{H}_{w}^{2} \odot \mathcal{H}_{w}^{2}\right)^{*}$.

We aim to relate Hankel forms and Volterra operators. The primitive of $f \in \mathcal{D}$ with constant term 0 is denoted by

$$
\partial^{-1} f(s):=-\int_{s}^{+\infty} f(u) d u
$$

We observe that

$$
H_{g}(f h)=f(+\infty) h(+\infty) g(+\infty)+\left\langle\partial^{-1}\left(f^{\prime} h\right), g\right\rangle_{\mathcal{H}_{w}^{2}}+\left\langle\partial^{-1}\left(f h^{\prime}\right), g\right\rangle_{\mathcal{H}_{w}^{2}} .
$$

The Banach space $\partial^{-1}\left(\partial \mathcal{H}_{w}^{2} \odot \mathcal{H}_{w}^{2}\right)$ is the completion of the space of Dirichlet series $F$ whose derivatives have a finite sum representation $F^{\prime}=\sum_{k} f_{k} h_{k}^{\prime}$, under the norm

$$
\|F\|_{\partial^{-1}\left(\partial \mathcal{H}_{w}^{2} \odot \mathcal{H}_{w}^{2}\right)}:=|F(+\infty)|+\sum_{k}\left\|f_{k}\right\|_{\mathcal{H}_{w}^{2}}\left\|h_{k}\right\|_{\mathcal{H}_{w}^{2}}
$$

where the infimum is taken over all finite representations. The product rule $(f g)^{\prime}=$ $f^{\prime} g+f g^{\prime}$ implies that

$$
\mathcal{H}_{w}^{2} \odot \mathcal{H}_{w}^{2} \subset \partial^{-1}\left(\partial \mathcal{H}_{w}^{2} \odot \mathcal{H}_{w}^{2}\right)
$$

and then

$$
\left(\partial^{-1}\left(\partial \mathcal{H}_{w}^{2} \odot \mathcal{H}_{w}^{2}\right)\right)^{*} \subset\left(\mathcal{H}_{w}^{2} \odot \mathcal{H}_{w}^{2}\right)^{*}
$$

It has been shown in [14] that, for the space $\mathcal{H}_{0}^{2}=\left\{f \in \mathcal{H}^{2}: f(+\infty)=0\right\}$, the inclusion $\left(\partial^{-1}\left(\partial \mathcal{H}_{0}^{2} \odot \mathcal{H}_{0}^{2}\right)\right)^{*} \subset\left(\mathcal{H}_{0}^{2} \odot \mathcal{H}_{0}^{2}\right)^{*}$ is strict. As for the space $\mathcal{H}_{w}^{2}$, the question whether the inclusion is strict remains open.

The membership of $g$ in $\left(\partial^{-1}\left(\partial \mathcal{H}_{w}^{2} \odot \mathcal{H}_{w}^{2}\right)\right)^{*}$ is equivalent to the boundedness of the so-called "half-Hankel form"

$$
(f, h) \mapsto\left\langle\partial^{-1}\left(f^{\prime} h\right), g\right\rangle_{\mathcal{H}_{w}^{2}} .
$$

As in the case of $\mathcal{H}^{2}$, the boundedness of $T_{g}$ implies the boundedness of $H_{g}$.

Theorem 5 If the Volterra operator $T_{g}$ is bounded on $\mathcal{H}_{w}^{2}$, then the Hankel form $H_{g}$ is bounded.

Proof We adapt the proof of Corollary 6.2 in [13] to the framework of the polydisk $\mathbb{D}^{\infty}$. Polarizing the Littlewood-Paley formula (1), we get

$$
\langle f, g\rangle_{\mathcal{H}_{w}^{2}}=f(+\infty) g(+\infty)+4 \int_{\mathbb{D}^{\infty}} \int_{\mathbb{R}} \int_{0}^{+\infty} f_{\chi}^{\prime}(\sigma+i t) \overline{g_{\chi}^{\prime}(\sigma+i t)} \sigma d \sigma \frac{d t}{1+t^{2}} d \mu_{w}(\chi)
$$


Then, we derive an expression of the half-Hankel form

$$
\left\langle\partial^{-1}\left(f^{\prime} h\right), g\right\rangle_{\mathcal{H}_{w}^{2}}=4 \int_{\mathbb{D}^{\infty}} \int_{\mathbb{R}} \int_{0}^{+\infty} f_{\chi}^{\prime}(\sigma+i t) h_{\chi}(\sigma+i t) \overline{g_{\chi}^{\prime}(\sigma+i t)} \sigma d \sigma \frac{d t}{1+t^{2}} d \mu_{w}(\chi)
$$

Since $T_{g}$ is bounded on $\mathcal{H}_{w}^{2}$, the Carleson measure characterization (4.1) induces that the form (8.3) is also bounded. Then $H_{g}$ is bounded on $\mathcal{H}_{w}^{2} \odot \mathcal{H}_{w}^{2}$ by the inclusion (8.2).

The previous Theorem states that we have

$$
\mathcal{X}_{w} \subset\left(\mathcal{H}_{w}^{2} \odot \mathcal{H}_{w}^{2}\right)^{*}
$$

The rest of the section is devoted to study the reverse inclusion.

Let $l_{w}^{2}$ denote the Hilbert space of complex sequences $a=\left(a_{n}\right)_{n}$ such that

$$
\|a\|_{l_{w}^{2}}:=\left(\sum_{n \geq 1} \frac{\left|a_{n}\right|^{2}}{w_{n}}\right)^{1 / 2}<\infty .
$$

A sequence $\left(\rho_{n}\right)_{n}$ generates the following multiplicative Hankel form

$$
\rho(a, b):=\sum_{n=1}^{+\infty} \sum_{m=1}^{+\infty} a_{m} b_{n} \frac{\rho_{m n}}{w_{m n}}, a, b \in l_{w}^{2} .
$$

The symbol of the form is the Dirichlet series $g(s)=\sum_{n \geq 1} \overline{\rho_{n}} n^{-s}$. The form $\rho$ is said to be bounded if there is a constant $C$ such that

$$
|\rho(a, b)| \leq C\|a\|_{l_{w}^{2}}\|b\|_{l_{w}^{2}} .
$$

If $f$ and $h$ are Dirichlet series with coefficients $a$ and $b$, respectively, we have

$$
H_{g}(f h)=\langle f h, g\rangle_{\mathcal{H}_{w}^{2}}=\rho(a, b)
$$

When the symbol $g$ has non negative coefficients, there is equivalence between the boundedness of $H_{g}$ and the half-Hankel form (8.3). In fact, the proof given for $\mathcal{H}^{2}$ in [14] is valid for the spaces $\mathcal{H}_{w}^{2}$.

Proposition 7 Let $g(s)=\sum_{n \geq 1} \overline{\rho_{n}} n^{-s}$ be in $\mathcal{H}_{w}^{2}$. The linear functional defined on $\mathcal{H}_{w}^{2}$

$$
v_{g}(f):=\langle f, g\rangle_{\mathcal{H}_{w}^{2}}
$$


is bounded on $\partial^{-1}\left(\partial \mathcal{H}_{w}^{2} \odot \mathcal{H}_{w}^{2}\right)$ if and only if the weighted form

$$
J_{g}(a, b)=\sum_{n=1}^{+\infty} \sum_{m=1}^{+\infty} a_{m} b_{n} \frac{\log n}{\log m+\log n} \frac{\rho_{m n}}{w_{m n}}
$$

(where it is understood that for $m=n=1$, the summand is 0 ) is bounded on $l_{w}^{2} \odot l_{w}^{2}$. The norms are equivalent, i.e.

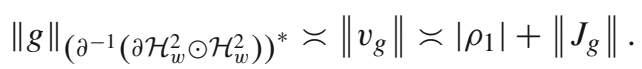

If $\rho_{k} \geq 0$ for all $k$, then $g \in\left(\partial^{-1}\left(\partial \mathcal{H}_{w}^{2} \odot \mathcal{H}_{w}^{2}\right)\right)^{*}$ if and only if $g \in\left(\mathcal{H}_{w}^{2} \odot \mathcal{H}_{w}^{2}\right)^{*}$, with equivalent norms.

Proposition 7 will enable us to provide examples of symbols $g$ for which the Hankel form $H_{g}$ and the half-Hankel form (8.3) are bounded, but the Volterra operator $T_{g}$ is unbounded (see the proof of Proposition 9). This differs from the case of weighted Dirichlet spaces on the unit disk, for which the boundedness of $H_{g}$, the form (8.3) and $T_{g}$ are equivalent [1].

For convergence reasons, we will consider Hankel forms defined on Dirichlet series without constant term. So we will work on the space

$$
\mathcal{H}_{w, 0}^{2}=\left\{f \in \mathcal{H}_{w}^{2}: f(+\infty)=0\right\}
$$

We have seen in Lemma 1 that the space $\mathcal{H}_{w}^{2}$ is embedded in a Bergman space of the form $A_{i, \delta}\left(\mathbb{C}_{1 / 2}\right)$. For $\delta>0$, it is thus natural to define the Hankel form

$$
H^{(\delta)}(f h):=\int_{1 / 2}^{+\infty} f(\sigma) h(\sigma)\left(\sigma-\frac{1}{2}\right)^{\delta} d \sigma, f, h \in \mathcal{H}_{w, 0}^{2}
$$

Such multiplicative forms have been considered in the context of $\mathcal{H}^{2}$ [12] and on $\mathcal{A}_{1}^{2}$ [9].

Since $K^{\mathcal{H}_{w}^{2}}(s, u)-1=\sum_{n \geq 2} w_{n} n^{-\bar{u}} n^{-s}$ is the reproducing kernel of $\mathcal{H}_{w, 0}^{2}$, we see that $H^{(\delta)}(f h)=\left\langle f h, \phi_{\delta}\right\rangle_{\mathcal{H}_{w}^{2}}$, where

$$
\phi_{\delta}(s)=\int_{1 / 2}^{+\infty}\left[K^{\mathcal{H}_{w}^{2}}(s, \sigma)-1\right]\left(\sigma-\frac{1}{2}\right)^{\delta} d \sigma=\sum_{n=2}^{+\infty} \frac{w_{n}}{\sqrt{n}(\log n)^{\delta+1}} n^{-s} .
$$

Proposition 8 Let $\delta>0$ as in (2.5). Then $H^{(\delta)}$ defined in (8.5) is a multiplicative Hankel form with symbol $\phi_{\delta}$, which is bounded on $\mathcal{H}_{w, 0}^{2} \odot \mathcal{H}_{w, 0}^{2}$. 
Proof The proof is similar to that of Theorem 13 in [9]. The Cauchy-Schwarz inequality ensures that

$$
\left|H^{(\delta)}(f h)\right| \leq\left(\int_{1 / 2}^{+\infty}|f(\sigma)|^{2}\left(\sigma-\frac{1}{2}\right)^{\delta} d \sigma\right)^{1 / 2}\left(\int_{1 / 2}^{+\infty}|h(\sigma)|^{2}\left(\sigma-\frac{1}{2}\right)^{\delta} d \sigma\right)^{1 / 2}
$$

If $f(s)=\sum_{n=2}^{+\infty} a_{n} n^{-s}$, notice the pointwise estimate

$$
|f(\sigma)|^{2} \leq\|f\|_{\mathcal{H}_{w}^{2}}^{2}\left(\sum_{n=2}^{+\infty} w_{n} n^{-2 \sigma}\right) \lesssim\|f\|_{\mathcal{H}_{w}^{2}}^{2} 4^{-\sigma}, \text { for } \sigma \geq 1
$$

Since the bounded measure $d \mu(\sigma+i t)=\chi)_{1 / 2,1]}(\sigma)\left(\sigma-\frac{1}{2}\right)^{\delta} d \sigma$, supported on the real line, is Carleson for $A_{i, \delta}\left(\mathbb{C}_{1 / 2}\right), \mu$ is Carleson for $\mathcal{H}_{w}^{2}$ by Lemma 6 , and

$$
\int_{1 / 2}^{+\infty}|f(\sigma)|^{2}\left(\sigma-\frac{1}{2}\right)^{\delta} d \sigma=\left(\int_{1 / 2}^{1}+\int_{1}^{+\infty}\right)|f(\sigma)|^{2}\left(\sigma-\frac{1}{2}\right)^{\delta} d \sigma \lesssim\|f\|_{\mathcal{H}_{w}^{2}}^{2} .
$$

We next exhibit symbols giving rise to bounded Hankel forms and bounded halfHankel forms, though the associated Volterra operator is unbounded.

Proposition 9 We have the strict inclusions

$$
\begin{gathered}
\mathcal{X}\left(\mathcal{H}_{w, 0}^{2}\right) \subset_{\neq}\left(\mathcal{H}_{w, 0}^{2} \odot \mathcal{H}_{w, 0}^{2}\right)^{*} ; \\
\mathcal{X}_{w} \subset_{\neq}\left(\mathcal{H}_{w}^{2} \odot \mathcal{H}_{w}^{2}\right)^{*}
\end{gathered}
$$

Proof It just remains to check the strictness of the inclusions. For the exponent $\delta=$ $\delta(w)$ and $\frac{1}{2} \leq a<1$, consider the symbol in $\mathcal{H}_{w, 0}^{2}$

$$
g(s)=\sum_{n=2}^{+\infty} \frac{w_{n}}{n^{a}(\log n)^{\delta+1}} n^{-s} .
$$

From Proposition 8 and the fact that the coefficients are positive, $g$ is in $\left(\mathcal{H}_{w, 0}^{2} \otimes \mathcal{H}_{w, 0}^{2}\right)^{*}$ for any $\frac{1}{2} \leq a<1$. In fact, the half Hankel form corresponding to $g$ is bounded. We have seen in Proposition 4 that $T_{g}$ is not bounded on $\mathcal{H}_{w}^{2}$. Since $T_{g} 1=g, g$ does not belong to $\mathcal{X}\left(\mathcal{H}_{w, 0}^{2}\right)$.

In order to prove that $g \in\left(\mathcal{H}_{w}^{2} \odot \mathcal{H}_{w}^{2}\right)^{*}$, we consider the associated multiplicative form $\rho$ (8.4). Let $f, h$ be Dirichlet series with coefficients $a, b$, belonging to $\mathcal{H}_{w}^{2}$. Since 


$$
\begin{aligned}
\rho(a, b) & =\sum_{m, n \geq 2} a_{m} b_{n} \frac{\rho_{m n}}{w_{m n}}+a_{1} \sum_{n=1}^{+\infty} b_{n} \frac{\rho_{n}}{w_{n}}+b_{1} \sum_{m=1}^{+\infty} a_{m} \frac{\rho_{m}}{w_{m}} \\
& =H_{g}((f-f(\infty))(g-g(\infty)))+f(\infty)\langle h, g\rangle_{\mathcal{H}_{w}^{2}}+g(\infty)\langle f, g\rangle_{\mathcal{H}_{w}^{2}},
\end{aligned}
$$

the first part of the proof entails that $H_{g}$ is bounded on $\mathcal{H}_{w}^{2} \odot \mathcal{H}_{w}^{2}$.

\section{$8.2 \mathcal{X}_{w}$ and the dual of $\mathcal{H}_{w}^{1}$}

Keeping in mind the results known for Bergman spaces of the unit disk, it is natural to compare $\mathcal{X}_{w}$ and $\left(\mathcal{H}_{w}^{1}\right)^{*}$.

In general, the dual of $\mathcal{H}_{w}^{1}$ is not known. However, it is shown in [9] that

$$
\mathcal{K} \subset\left(\mathcal{A}_{1}^{1}\right)^{*}
$$

where $\mathcal{K}$ is the space of Dirichlet series $f(s)=\sum_{n=1}^{+\infty} a_{n} n^{-s}$ such that

$$
\sum_{n=1}^{+\infty} \frac{d_{4}(n)}{[d(n)]^{2}}\left|a_{n}\right|^{2}<\infty .
$$

The following consequence of this inclusion will stress upon the difference between the finite and infinite dimensional setting.

Proposition $10\left(\mathcal{A}_{1}^{1}\right)^{*}$ is not contained in $\mathcal{X}\left(\mathcal{A}_{1}^{2}\right)$.

Proof By Abel summation and the Chebyshev estimate, the symbol

$$
g(s)=\sum_{n=2}^{+\infty} \frac{d(n)}{n^{a}(\log n)^{2}} n^{-s}, \text { for } \frac{1}{2}<a<1,
$$

is in $\mathcal{K}$, and thus in $\left(\mathcal{A}_{1}^{1}\right)^{*}$. However, $T_{g}$ is unbounded on $\mathcal{A}_{1}^{2}$ (Proposition 4).

\section{3 $\mathcal{X}_{w}$ and the spaces $\mathcal{H}_{w}^{p}$}

It has been shown in [13] that $B M O A\left(\mathbb{C}_{0}\right) \cap \mathcal{D} \subset_{\neq} \mathcal{X}\left(\mathcal{H}^{2}\right) \subset_{\neq} \cap_{0<p<\infty} \mathcal{H}^{p}$. We have an analogue for Bergman spaces of Dirichlet series.

Theorem 6 We have the strict inclusions

$$
\operatorname{BMOA}\left(\mathbb{C}_{0}\right) \cap \mathcal{D} \subset_{\neq} \mathcal{X}_{w} \subset_{\neq} \cap_{0<p<\infty} \mathcal{H}_{w}^{p}
$$

Proof The inclusions have been proved in Theorem 1 and Corollary 1. As observed in [13], the symbols $g(s)=\sum_{n=2}^{+\infty} \frac{\psi(n)}{\log n} n^{-s}$, where $\psi$ is the completely multiplicative function defined on the primes by $\psi(p):=\lambda p^{-1} \log p, 0<\lambda \leq 1$, are in $\mathcal{X}\left(\mathcal{H}^{2}\right)$, and satisfy 


$$
\sum_{n=1}^{+\infty} \psi(n) n^{-\sigma} \asymp \exp \left(\lambda \sum_{p} \frac{\log p}{p^{1+\sigma}}\right) \asymp \exp \left(\lambda \frac{1}{\sigma}\right), \sigma>0
$$

Hence, they are not in $B M O A\left(\mathbb{C}_{0}\right)$, though they belong to $\mathcal{X}_{w}$ (Lemma 9).

The second inclusion is strict by Proposition 6.

With the method of Proposition 4, one can show that $g(s)=\sum_{n \geq 2} \frac{n^{-a}}{\log n} n^{-s}, 1 / 2 \leq$ $a<1$, is not in $\mathcal{X}_{w}$, though it belongs to $B M O A\left(\mathbb{C}_{1-a}\right)$ [13]. Therefore, we have the strict inclusion

$$
\mathcal{X}_{w} \subset_{\neq} \operatorname{Bloch}\left(\mathbb{C}_{1 / 2}\right)
$$

\section{4 $\mathcal{X}_{w} \cap \mathcal{D}_{d}$ and Bloch spaces}

Theorem 7 Let d be a positive integer. The following inclusions hold

$$
\mathcal{D}_{d} \cap \operatorname{Bloch}\left(\mathbb{C}_{0}\right) \subset \mathcal{D}_{d} \cap \mathcal{X}_{w} \subset \neq \mathcal{B}^{-1} \operatorname{Bloch}\left(\mathbb{D}^{d}\right)
$$

Proof The first inclusion has been shown in Theorem 1(a).

If $g$ is in $\mathcal{D}_{d} \cap \mathcal{X}_{w}$, Theorem 5 implies that $H_{g}$ is bounded on $\mathcal{H}_{w}^{2}$. Therefore, the form $H_{\mathcal{B} g}(1.4)$ is bounded on the Bergman space $H_{w}^{2}\left(\mathbb{D}^{d}\right)$. From [17], $\mathcal{B} g$ is in $\operatorname{Bloch}\left(\mathbb{D}^{d}\right)$.

Here is a function $g$ which is not in $\mathcal{X}_{w}$, such that $\mathcal{B} g$ is in Bloch $\left(\mathbb{D}^{2}\right)$. Suppose that

$$
g^{\prime}(s)=\frac{1}{1-2^{-s}} \log \left(\frac{1}{1-3^{-s}}\right), s \in \mathbb{C}_{0} .
$$

Straightforward computations show that $\mathcal{B} g \in \operatorname{Bloch}\left(\mathbb{D}^{2}\right)$. The norms $\|\cdot\|_{A_{\beta}^{2}\left(\mathbb{D}^{2}\right)}$ and $\|\cdot\|_{B_{\beta}^{2}\left(\mathbb{D}^{2}\right)}$ being equivalent, our setting will be the space $A_{\beta}^{2}\left(\mathbb{D}^{2}\right)$. Now, for

$$
F(z)=\sum_{n=1}^{\infty} \frac{(n+1)^{\frac{\beta-1}{2}}}{\log (n+1)} z^{n}=\sum_{n=0}^{\infty} a_{n} z^{n}, z \in \mathbb{D}
$$

define $f(s)=F\left(2^{-s}\right) F\left(3^{-s}\right)$, for $s \in \mathbb{C}_{0}$. We have

$$
\|f\|_{\mathcal{H}_{w}^{2}}^{2}=\|F\|_{A_{\beta}^{2}(\mathbb{D})}^{4} \asymp\left(\sum_{n=1}^{\infty} \frac{1}{(n+1)(\log (n+1))^{2}}\right)^{2}<\infty .
$$

Putting

$$
h_{1}\left(z_{1}\right)=F\left(z_{1}\right) \frac{1}{1-z_{1}}=\sum_{m=0}^{\infty} A_{m} z_{1}^{m}, z_{1} \in \mathbb{D} \text {, }
$$




$$
h_{2}\left(z_{2}\right)=F\left(z_{2}\right) \log \left(\frac{1}{1-z_{2}}\right)=\sum_{n=0}^{\infty} B_{n} z_{2}^{n}, z_{2} \in \mathbb{D} \text {, }
$$

we have $A_{m} \gtrsim \frac{(m+1)^{\frac{\beta+1}{2}}}{\log (m+1)}$ and $B_{n} \gtrsim(n+1)^{\frac{\beta-1}{2}}$. Therefore,

$$
\begin{aligned}
\left\|T_{g} f\right\|_{\mathcal{H}_{w}^{2}}^{2} & =\left\|R^{-1}\left(h_{1} h_{2}\right)\right\|_{A_{\beta}^{2}\left(\mathbb{D}^{2}\right)}^{2} \asymp \sum_{m, n \geq 1} \frac{\left|A_{m}\right|^{2}\left|B_{n}\right|^{2}}{(m+n+1)^{2}(m+1)^{\beta}(n+1)^{\beta}} \\
& \gtrsim \sum_{m \geq 1} \frac{m+1}{(\log (m+1))^{2}} \frac{\log (m+1)}{(m+1)^{2}}=\sum_{m \geq 1} \frac{1}{(m+1) \log (m+1)}=+\infty,
\end{aligned}
$$

which proves the claim.

A consequence of Theorems 1 and 6 is that

$$
\operatorname{Bloch}\left(\mathbb{C}_{0}\right) \cap \mathcal{D}_{d} \subset \cap_{0<p<\infty} \mathcal{H}_{d, w}^{p}
$$

This inclusion can be viewed as a counterpart of the situation of the disk, where $\operatorname{Bloch}(\mathbb{D}) \subset \cap_{0<p<\infty} A_{\beta}^{p}(\mathbb{D})$.

Acknowledgements Open access funding provided by Austrian Science Fund (FWF).

Open Access This article is licensed under a Creative Commons Attribution 4.0 International License, which permits use, sharing, adaptation, distribution and reproduction in any medium or format, as long as you give appropriate credit to the original author(s) and the source, provide a link to the Creative Commons licence, and indicate if changes were made. The images or other third party material in this article are included in the article's Creative Commons licence, unless indicated otherwise in a credit line to the material. If material is not included in the article's Creative Commons licence and your intended use is not permitted by statutory regulation or exceeds the permitted use, you will need to obtain permission directly from the copyright holder. To view a copy of this licence, visit http://creativecommons.org/licenses/by/4.0/.

\section{References}

1. Aleman, A., Perfekt, K.M.: Hankel forms and embedding theorems in weighted Dirichlet spaces. Int. Math. Res. Not. IMRN 19, 4435-4448 (2012)

2. Aleman, A., Siskakis, A.G.: Integration operators on Bergman spaces. Indiana Univ. Math. J. 46, 337-356 (1997)

3. Anderson, J., Clunie, J., Pommerenke, C.: On Bloch functions and normal functions. J. Reine Angew. Math. 270, 12-37 (1974)

4. Apostol, T.M.: Introduction to Analytic Number Theory. Springer, New-York (1976)

5. Bailleul, M., Brevig, O.F.: Composition operators on Bohr-Bergman spaces of Dirichlet series. Ann. Acad. Sci. Fen. M. 41, 129-142 (2016)

6. Bailleul, M., Lefèvre, P.: Some Banach spaces of Dirichlet series. Stud. Math. 226(1), 17-55 (2015)

7. Bayart, F.: Compact composition operators on a Hilbert space of Dirichlet series. Ill. J. Math. 47(3), 725-743 (2003)

8. Bayart, F., Brevig, O.F.: Composition operators and embeddings theorems for some function spaces of Dirichlet series. Math. Z. 293(3-4), 989-1014 (2019)

9. Bayart, F., Brevig, O.F., Haimi, A., Ortega-Cerda, J., Perfekt, K.M.: Contractive inequalities for Bergman spaces and multiplicaive Hankel forms. Trans. Am. Math. Soc. 371(1), 681-707 (2019) 
10. Bayart, F., Queffélec, H., Seip, K.: Approximation numbers of composition operators on $H^{p}$ spaces of Dirichlet series. Ann. Inst. Fourier (Grenoble) 66(2), 551-588 (2016)

11. Bohr, H.: Über die Bedeutung der Potenzreihen unendlich vieler Variabeln in der Theorie der Dirichletschen reihen $\sum a_{n} / n^{s}$. Nachr. Ges. Wiss. Göttingen Math. Phys. K1. 1913, 441-488 (1913)

12. Brevig, O.F., Perfekt, K.-M., Seip, K., Siskakis, A.G., Vukotic, D.: The multiplicative Hilbert matrix. Adv. Math. 302, 410-432 (2016)

13. Brevig, O.F., Perfekt, K.-M., Seip, K.: Volterra operators on Hardy spaces of Dirichlet series. J. Reine Angew. Math. (2019). https://doi.org/10.1515/crelle-2016-0069

14. Brevig, O.F., Perfekt, K.-M.: Weak product of Dirichlet series. Integral Equ. Oper. Theory 86(4), 453-473 (2016)

15. Cima, J.A., Schober, G.: Analytic functions with bounded mean oscillation and logarithms of $H^{p}$ functions. Math. Z. 151, 295-300 (1976)

16. Cole, B.J., Gamelin, T.W.: Representing measures and Hardy spaces for the infinite polydisk algebra. Proc. Lond. Math. Soc. 3(53), 112-142 (1986)

17. Constantin, O.: Weak product decompositions and Hankel operators on vector-valued Bergman spaces. J. Oper. Theory 59, 157-178 (2008)

18. Constantin, O.: Carleson embeddings and some classes of operators on weighted Bergman spaces. J. Math. Anal. Appl. 365, 668-682 (2010)

19. Hedenmalm, H., Lindqvist, P., Seip, K.: A Hilbert space of Dirichlet series and systems of dilated functions in $L_{2}(0 ; 1)$. Duke Math. J. 86, 1-37 (1997)

20. Ivic, A.: The Riemann Zeta-Function, Theory and Applications. Dover Publications Inc., New York (2003)

21. McCarthy, J.: Hilbert spaces of Dirichlet series and their multipliers. Trans. Am. Math. Soc. 356, 881-893 (2004)

22. Olevskii, A.M.: Fourier Series with Respect to General Orthonormal Systems. Springer, Berlin (1975)

23. Olsen, J.F.: Local properties of Hilbert spaces of Dirichlet series. J. Funct. Anal. 261, 2669-2696 (2011)

24. Olsen, J.F., Saksman, E.: On the boundary behavior of the Hardy space of Dirichlet series and a frzme bound estimate. J. Reine Angew. Math. 663, 33-66 (2012)

25. Olsen, J.F., Seip, K.: Local interpolation in Hilbert spaces of Dirichlet series. Proc. Am. Math. Soc. 136, 203-212 (2008)

26. Pommerenke, C.: Schlichte Funktionen und analytische Funktionen von beschränkter mittlerer Oscillation. Comment. Math. Helv. 52(4), 591-602 (1977)

27. Seip, K.: Zeros of functions in Hilbert spaces of Dirichlet series. Math. Z. 274(3-4), 1327-1339 (2013)

28. Smith, W.: Composition operators between Bergman spaces and Hardy spaces. Trans. Am. Math. Soc. 348, 2331-2348 (2013)

29. Stanton, C.S.: Counting functions and majorization for Jensen measures. Pac. J. Math. 125, 459-468 (1986)

30. Wilson, B.M.: Proofs of some formulae enunciated by Ramanujan. Proc. Lond. Math. Soc. 2(1), 235255 (1923)

31. Zhu, K.: Operator Theory in Function Spaces. Mathematical Surveys and Monographs, vol. 138, 2nd edn. American Mathematical Society, Providence (2007)

Publisher's Note Springer Nature remains neutral with regard to jurisdictional claims in published maps and institutional affiliations. 